Article

\title{
Numerical Simulation and Analysis of Fish-Like Robots Swarm
}

\author{
Shuman $\mathrm{Li}^{1}{ }^{1}$, Chao $\mathrm{Li}^{1}{ }^{1}$, Liyang $\mathrm{Xu}{ }^{1}$, Wenjing Yang ${ }^{1, *}$ and Xucan Chen ${ }^{2}$ \\ 1 State Key Laboratory of High Performance Computing, College of Computer, \\ National University of Defense Technology, Changsha 410073, China; lishuman13@nudt.edu.cn (S.L.); \\ dirk911@nudt.edu.cn (C.L.); xuliyang08@nudt.edu.cn (L.X.) \\ 2 National Innovation Institute of Defense Technology (NIIDT), Beijing 100060, China; 13973162892@139.com \\ * Correspondence: wenjing.yang@nudt.edu.cn; Tel.: +86-158-0256-9718
}

Received: 25 March 2019; Accepted: 16 April 2019; Published: 21 April 2019

check for updates

\begin{abstract}
Artificial fish-like robot is an important branch of underwater robot research. At present, most of fish-like robot research focuses on single robot mechanism behavior, some research pays attention to the influence of the hydro-environment on robot crowds but does not reach a unified conclusion on the efficiency of fish-like robots swarm. In this work, the fish-like robots swarm is studied by numerical simulation. Four different formations, including the tandem, the phalanx, the diamond, and the rectangle are conducted by changing the spacing between fishes. The results show that at close spacing, the fish in the back can obtain a large wake from the front fish, but suffers large lateral power loss from the lateral fish. On the contrary, when the spacing is large, both the wake and pressure caused by the front and side fishes become small. In terms of the average swimming efficiency of fish swarms, we find that when the fish spacing is less than $1.25 \mathrm{~L}$ ( $L$ is the length of the fish body), the tandem swarm is the best choice. When the spacing is $1.25 \mathrm{~L}$, the tandem, diamond and rectangle swarms have similar efficiency. When the spacing is larger than $1.25 \mathrm{~L}$, the rectangle swarm is more efficient than other formations. The findings will provide significant guidance for the control of fish-like robots swarm.
\end{abstract}

Keywords: fish schooling; hydrodynamics; swimming efficiency; robots swarm; robot controlling

\section{Introduction}

The multi-robot control has been a topic of broad interest in recent years. A single robot with multiple capabilities cannot necessarily accomplish an intended job, whereas robots in a robot swarm in which each robot with its own functions, can be more flexible, robust, cost-effective and do complex tasks [1]. Some literature [2-4] focuses on the collaborative multi-robot studies, including the coordination and control techniques, the communication among robot groups, mapping and localization as well as the architecture of the multi-robot system. Other studies [5,6] are concerned with certain collaborative missions, such as box-pushing, formatting and rescue. In addition, some studies $[7,8]$ focus on heterogeneous robots swarm which is capable of performing user-defined tasks. Based on the analysis of existing robots swarm systems [1], researchers designed the hardware architecture $[9,10]$ and software environment [11] of robot swarms, which enables land robots to handle various tasks in unknown or dynamic environments. Formation control is also a research direction of swarm robots [12-15]. Underwater robot swarms, unlike air or land robots, can hardly maintain their formation through communication [16]. Besides, in the underwater environment, the velocity of fluids, viscosity of fluids, the complex geometry environment condition and even the interaction between robots can change the underwater robots swarm behaviors. Therefore, it is necessary to figure out a stable and efficient formation of underwater robots swarm. 
At present, many researchers use numerical simulation methods to study underwater robots, avoiding the limitations of experimental conditions. Furthermore, this method can provide a more intuitive hydrodynamic phenomenon, which can provide guidance for the shape design and control of the underwater robots [17-19]. There is evidence to show that fish-like underwater robots have better motion ability [20-23]. The research on the formation of underwater robots is mostly based on fish-like robots.

Weihs [24] proposes a hydrodynamic theory of schooling that a fish in a diamond school swimming between two adjacent fish wakes can save energy. Novati et al. [25], through employing reinforcement learning, adjust swimming motions and relative positions to reach a stable tandem configuration, at the same time the follower swimmer can reduce energy. Maertens et al. [26] establish optimal undulatory propulsion for a single fish by numerical simulation and find that the follower fish saved energy at any position as it can appropriately modulate its body motions according to the oncoming vortices. Ashraf et al. [27] show that the phalanx pattern of swimming animals can be a strategy to optimize swimming performance. Verma et al. [28] discuss several kinds of coordinated swimming patterns and the results show that through deep reinforcement learning autonomous swimmers can exploit unsteady flow fields to swim efficiently. Dai at al. [29] find that multiple kinds of fish school formations can keep stable by hydrodynamic interactions alone.

The above studies provide many good ideas and algorithms for the control of fish-like robots swarm and propose some efficient fish-like robot formations. However, these studies adopt different geometric models, kinematic models, and hydrological environments, but do not reach a unified conclusion on the efficiency of fish-like robots swarm. Thus, it is difficult for them to provide much guidance on how to select the efficient fish-like robot formation configuration under specific conditions. Therefore, this paper studies the influence of changing swarm configuration on multiple fish-like robots based on the same geometric model, kinematic model, and hydrological environments. To be specific, the main contributions are summarized as follows:

- Efficiency savings are simulated by changing the formation of the fish-like robots swarm and the spacing between fishes. The results show that the efficiency of the tandem swarm is high at close spacing, and the efficiency of the rectangle swarm is high at large spacing.

- Set the hydro-environment to a low Re number [30] and simple geometry boundary condition, which is closer to the gentle aquatic environment. Set four kinds of fish-like robots swarms: the tandem, the phalanx, the diamond and the rectangle, and the spacing between fishes is changed to test the thrust coefficient, the lateral power loss coefficient and the Froude efficiency of fishes.

- Discuss the influence of the wake $[25,31]$ and the pressure field generated by the fish-like robot tail swing on the Froude efficiency, and analyze the effect of fish-like robot spacing on swarm performance. The influence of the wake especially the direction and size on tandem swarm is analyzed detailedly, and the results of other formations are consistent with it. The findings will provide significant guidance for the control of fish-like robots swarm.

Based on the open-source software OpenFOAM [32-34], this work models and simulates fish-like robots, and moves mesh with a parallel dynamic mesh method based on the radial basis $[35,36]$.

The rest of the paper is organized as follows. Section 2 describes the computational methods, and includes the fish-like robot geometric and kinematic model, the computational fluid dynamics numerical method, the fish-like robots swarm configuration and the performance measurements. In Section 3, massive simulation results are presented and analyzed. Conclusions are drawn in the final section.

\section{Computational Methods}

\subsection{Geometrical Model and Kinematic Model}

The present study of fish-like robot swarms was based on two-dimensional simulations. For convenience, the fish-like robot is abbreviated as fish. In our work, we used a cross-section of the 
three-dimensional fish body that is in the work of Kern and Koumoutsakos [37] as the two-dimensional simulation model. The half outline of the two-dimensional fish body was described by $w(s)$, which is defined as the analytical functions of the arc length $s$ (measured from the nose) along the midline of the body. The analytical description of $w(s)$ is divided into three regions:

$$
w(s)=\left\{\begin{array}{lc}
\sqrt{2 w_{h} s-s^{2}} & 0 \leq s<s_{h} \\
w_{h}-\left(w_{h}-w_{t}\right)\left(\frac{s-s_{h}}{s_{t}-s_{h}}\right)^{2} & s_{h} \leq s<s_{t} \\
w_{t}(L-s) /\left(L-s_{t}\right) & s_{t} \leq s \leq L,
\end{array}\right.
$$

where $L$ is the body length, $w_{h}=s_{h}=0.04 L, w_{t}=0.01 L$ and $s_{t}=0.95 L$. Figure 1 a shows the half outline of the straight body.

Generally, the kinematics for the anguilliform swimmers can be considered as a backward travelling wave, with the amplitude increasing almost linearly from the head to the tail of the fish [38]. Here we adopted the equation specified by Carling [39] to describe the deforming motion of the fish body. The lateral undulation of the midline $y(s, t)$ is given as follows:

$$
y(s, t)=0.125 \frac{s / L+0.03125}{1.03125} \sin \left(2 \pi\left(\frac{s}{L}-\frac{t}{T}\right)\right)
$$

where $T$ is the undulation period. Controlled by this equation, the wavelength of the body undulations was $L$, and the maximum lateral excursion of the tail was $0.125 \mathrm{~L}$.

(a)

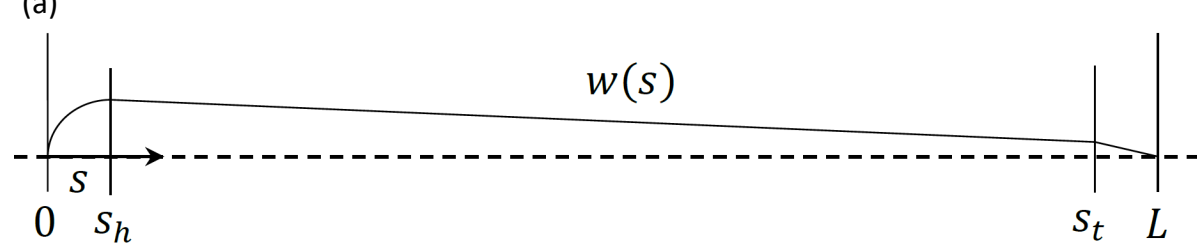

(b)

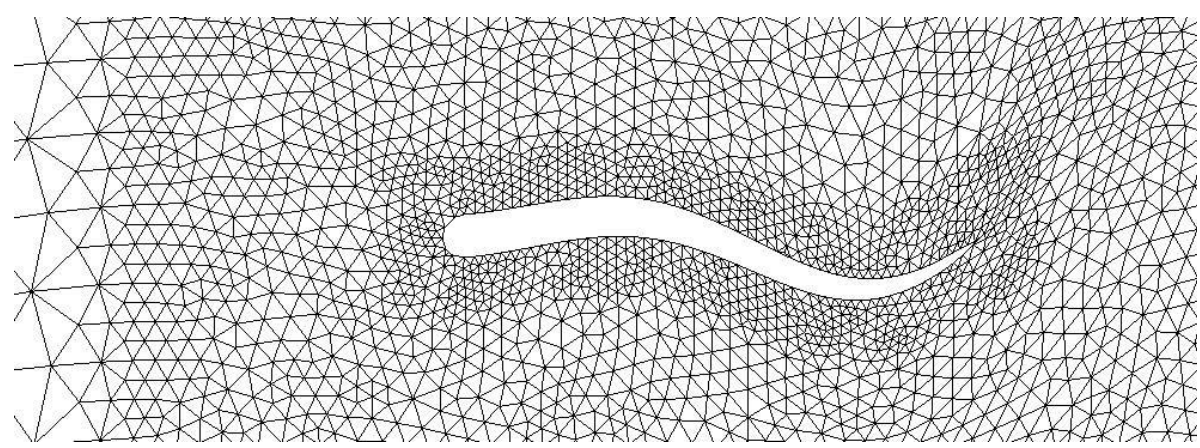

Figure 1. Illustration of the analytical description of the fish-like robot body. (a) Half outline of the body; (b) mesh distribution around the body.

\subsection{Numerical Method}

This work presents an arbitrary-Lagrangian-Eulerian(ALE) method [40-42] to simulate fish moving in the fluid. The governing equations of ALE formulation for the two-dimensional viscous incompressible flow are expressed as follows:

$$
\begin{gathered}
\nabla \cdot \boldsymbol{u}=\mathbf{0} \\
\frac{\partial \boldsymbol{u}}{\partial t}+\boldsymbol{c} \cdot \nabla \boldsymbol{u}=-\frac{\nabla p}{\rho}+v \nabla^{2} \boldsymbol{u}+\boldsymbol{b} .
\end{gathered}
$$

In these equations, $\rho$ and $v$ denote the density and the kinematic viscosity of the fluid respectively; $p$ and $\boldsymbol{u}$ represent the pressure and velocity of the flow respectively; $\boldsymbol{b}$ is the body force per unit mass, 
$c$ is the convective velocity which represents the relative velocity between the moving mesh and the fluid. The boundary conditions are set as follows: uniform velocity at the inlet, constant static pressure and zero-gradient velocity at the outlet, slip wall at the lateral boundaries, and no-slip wall on the surface of the cylinder. The interaction of the fish and the fluid is realized by setting a no-slip boundary condition on the robot surface and introducing a relation:

$$
\boldsymbol{u}_{b}=\boldsymbol{u}_{f},
$$

where $\boldsymbol{u}_{b}$ is the fish body velocity, $\boldsymbol{u}_{f}$ is the fluid velocity at the surface of fish body.

The governing equations were discretized by a finite volume approach with the second-order backward scheme in time and second-order liner scheme in space. The pressure-implicit split-operator (PISO) algorithm [43] was used to solve Equations (3) and (4) for velocity and pressure. The whole computational domain was decomposed by triangle mesh, as illustrated in Figure $1 \mathrm{~b}$. As the fields around the undulating fish may had a large gradient, mesh near the robot was refined with high resolution. The surface of the fish body was composed of 110-160 cells. All the simulations were based on the mesh scale from $5 \times 10^{4}-9 \times 10^{4}$ cells which has been tested to be large enough for our two-dimensional simulations. We used a parallel dynamic mesh method based on the radial basis function to move mesh on OpenFOAM.

\subsection{Swarm Configurations}

In this work, we mainly compared four different swarm configurations: tandem, phalanx, diamond and rectangle. For the tandem shape swarm in Figure 2a, fishes were swimming in a line with same interval spacing $d x$. In this configuration, the robot swimming in the front generated flow in the wake, thus the fish swimming downstream was affected by the unsteady flow. For the phalanx configuration in Figure $2 b$, fishes were placed in a column with spacing $d y$, and the effect of the lateral flow oscillating was considered. To synthetically take into account the two directional effects, we also designed the diamond swarm and the rectangle swarm: the diamond swarm was shaped by four fishes as shown in Figure 2c, and the rectangle swarm is shaped by four fishes as shown in Figure $2 \mathrm{~d}$. In these two configurations, the longitudinal spacing $d x$ and the lateral spacing $d y$ were set the same. The number of each fish in the swarm was also given in the Figure 2.

For all the swarm configurations, fishes were swimming in the same phase difference. We studied the hydrodynamic consequences with the spacing $d x$ and $d y$ varying from $0.5 L$ to $2 L$.

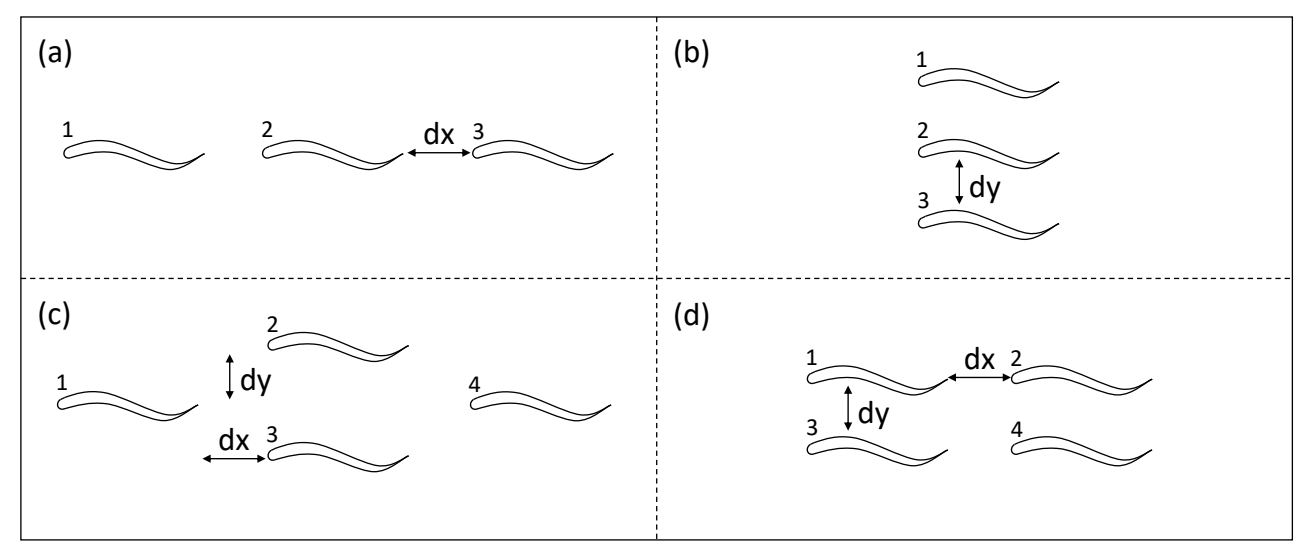

Figure 2. Graphical representation of the swarm configurations: (a) tandem, (b) phalanx, (c) diamond and (d) rectangle. Lateral spacing between neighbours is given by $d y$ and longitudinal spacing by $d x$. 


\subsection{Performance Measurements}

Steady undulatory swimming is characterized by two non-dimensional parameters, the flow Reynolds number $R e$ and the Strouhal number $S t$, which can be defined as follows:

$$
\begin{aligned}
& R e=\frac{U L}{v}, \\
& S t=\frac{f A}{U},
\end{aligned}
$$

where $U$ is the steady swimming speed, $v$ is the kinematic viscosity of the fluid, $f$ is the tail-beat frequency and $A$ is two times the lateral excursion of the tail tip. For the reason to introduce the viscous effect, we set the Reynolds number at a relatively low value $\left(1 \times 10^{3}\right)$ [30]. In order to obtain a constant swimming speed $U$, we attach the fish to a rigid tether at a given constant inlet velocity $U$. As the $R e$ and $U$ is set constant throughout the whole simulations, we vary the $S t$ only by setting different values of the tail-beat frequency.

The work of Borazjani and Sotiropoulos [44,45] proposes the method that can change the St whilst keeping constant swimming speed $U$ and Reynolds number $R e$. The flow induced by a model fish is simulated. The model fish is attached to and dragged by a rigid tether that translates the fish in a stationary fluid at a given constant velocity $U$. The desired value of $R e$ can be obtained by a fixed speed of the tether $U$. We can adjust the $S t$ through modifying the beating frequency of the fish tail $f$, and the fish always undulates its tail at the required constant frequency. The force acting on the fish body by the water flow is set to be $F$. If $F \neq 0$, the hypothetical tether absorbs the surplus force. Therefore, the net force exerting on the fish is zero, and it meets the constant swimming velocity assumption. In these cases, if the assumed tether is cut off instantaneously, the fish will accelerate or decelerate under the influence of the extra force $F$. For a given $R e$, the $S t$ is changed until the net average force that exerted on the fish is zero, i.e., $F=0$. In this case, the assumed tether clearly has no effect on the fish. This is because if the hypothetical tether is cut off, the fish will also keep swimming at a constant speed $U$. The fishes can follow this procedure to keep formation configurations and simulate natural "free swimming" conditions.

In other words, the simulations use "pinned" formation configurations for the simulated fishes, where the fishes are not allowed to deviate from their assigned positions, and an inflow velocity is imposed. The tail-beat frequency is then adjusted until the average thrust is approximately equal to the average drag, which is necessary to emulate the natural "free-swimming" condition.

It is still controversial and ambiguous to measure the swimming efficiency in numerical simulations. The Froude efficiency that indicates the proportion of the useful power in the total power is a relatively accurate and reasonable parameter, which is defined as:

$$
\eta=\frac{\bar{T} U}{\bar{T} U+{\overline{P_{y}}}^{\prime}}
$$

where $\bar{T}$ is the average thrust, $U$ is the swimming speed, and $\overline{P_{y}}$ is the average power loss due to the lateral undulations. To obtain the Froude efficiency during the steady swimming, the instantaneous thrust force and lateral power should be calculated first.

In our simulations, the fishes were placed in the middle of the computational domain and swim steadily along the $x$ direction. Here the fluid force along the $x$ direction can be computed by integrating the pressure and viscous forces on the fish body [44]:

$$
F(t)=\int_{S}\left(p n_{x}-\tau_{x j} n_{j}\right) d S
$$


where $n_{j}$ is the $j$-th component of the unit normal vector on $d S, \tau$ is the viscous stress tensor, $S$ is the surface of the body. To separate the contributions of thrust $T(t)$ and drag $D(t)$, we decompose the net force depending on whether $F(t)$ is positive or negative:

$$
\begin{gathered}
T(t)=\frac{1}{2} F(t)+\frac{1}{2} \int_{S}\left(\left|p n_{x}\right|+\left|\tau_{x j} n_{j}\right|\right) d S, \\
-D(t)=\frac{1}{2} F(t)-\frac{1}{2} \int_{S}\left(\left|p n_{x}\right|+\left|\tau_{x j} n_{j}\right|\right) d S .
\end{gathered}
$$

The swimming power was mainly consumed in the lateral direction, which can be calculated as follows:

$$
P_{y}=\int_{S}\left(p n_{y}-\tau_{y j} n_{j}\right) u_{y} d S,
$$

where $u_{y}$ is the lateral component of fish velocity. The non-dimensional thrust and lateral power loss coefficients are defined as:

$$
\begin{aligned}
& C_{t}=\frac{|T(t)|}{0.5 \rho U^{2} L^{2}}, \\
& C_{p}=\frac{\left|P_{y}\right|}{0.5 \rho U^{3} L^{2}} .
\end{aligned}
$$

We will also discuss the relation between wake flow and the Froude efficiency.

\section{Results and Discussion}

\subsection{Correctness Validation}

To approach the stable hydro-environment, we choose laminar flows, and the fluid velocity is $1 L / s$ and the Re number is 1000 . We did some work to ensure grid- and time-independence at the $R e$ number and the correctness of the simulation method.

In order to evaluate the grid dependence of the solutions, we built the coarse and fine unstructured grids that produce $M 1(33,399 \mathrm{cells}), \mathrm{M} 2(70,553 \mathrm{cells})$ and $M 3(191181 \mathrm{cells}$ ), respectively. The grid-independence analysis is performed by comparing the thrust of single fish obtained with the three meshes. As shown in Figure 3, the thrust of the single fish obtained with $M 2$ and $M 3$ are almost identical and the maximum relative difference is less than $1 \%$. Thus, a simulation of time-independence was achieved with M2. The same mesh scale as $M 2$ was adopted in the following simulations of fishes swarm.

In order to evaluate the time dependence of the solutions, we built the small and big $\Delta T$, and set $\Delta T 1(0.001), \Delta T 2(0.0001)$ and $\Delta T 3(0.00001)$, respectively. The time-independence analysis was performed by comparing the thrust of single fish obtained with the three $\Delta T$. As shown in Figure 4 , the thrust of the single fish obtained with all $\Delta T$ were almost identical. $\Delta T 2$ is adopted in the following simulations.

In order to evaluate the correctness of simulation method, we simulated a single fish under conditions in Carling [39], and the results are shown in Figure 5b. In Figure 5, Carling's model and results are presented on the left and ours on the right. Because of the difference of geometric models, the asymptotic mean velocity of fish and the convergence time were different, but the overall trend was consistent. We found that Hieber et al. [46] used the same geometric model as we did, and their asymptotic mean velocity was very close to our result. Therefore, we have reason to believe that our method is correct and the method is adopted in the following simulations. 


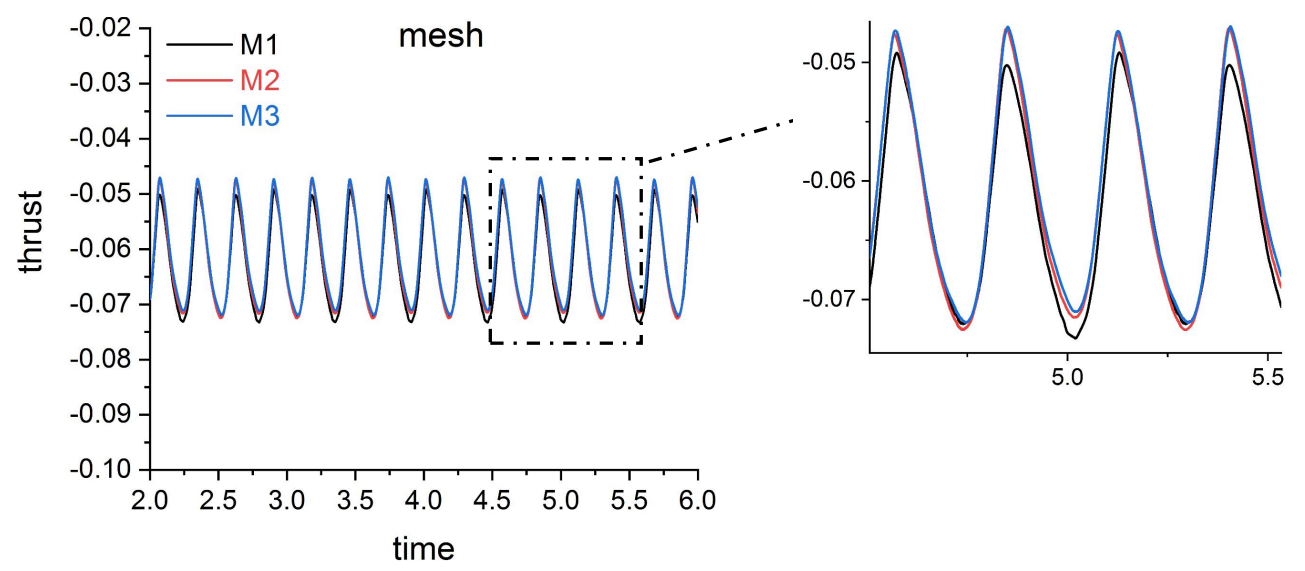

Figure 3. Result of grid-independence analysis.

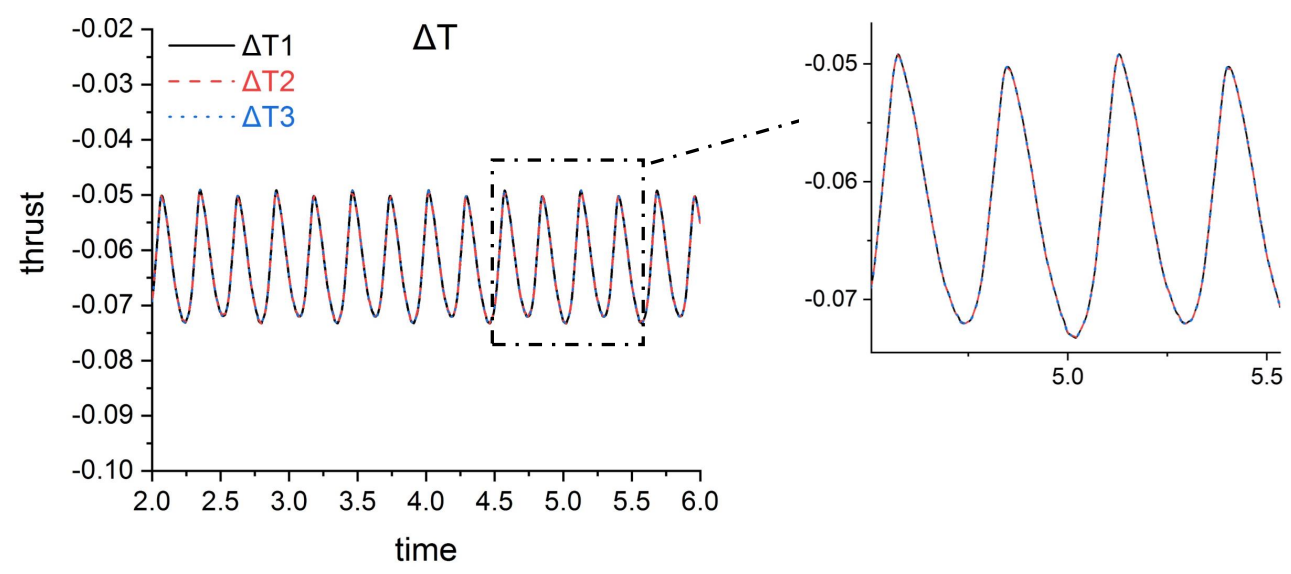

Figure 4. Result of time-independence analysis.
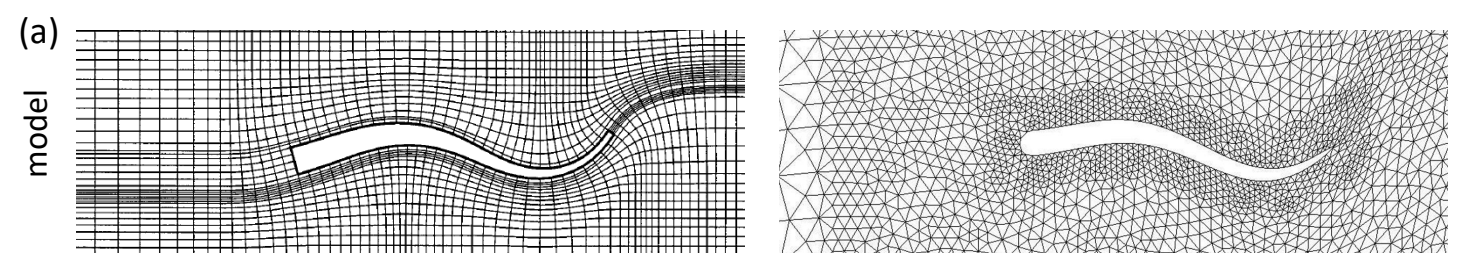

(b)
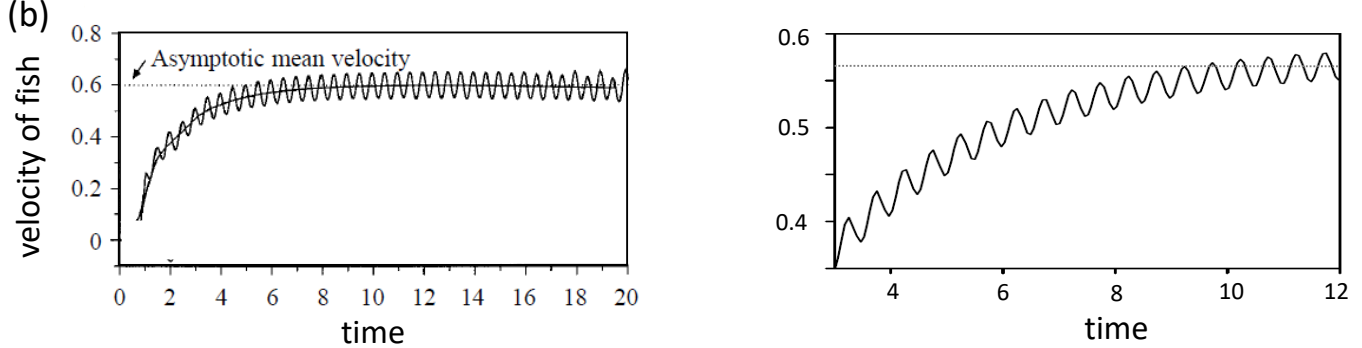

Figure 5. Result of method correctness analysis: (a) model, (b) image of velocity change with time.

In addition, it is important to note that only when the net force of the fish body is zero, the fishes are able to maintain formation configurations and the Froude efficiency is useful [44,45]. If the net force was not zero, the thrust force and the drag force were not equal, which means the fish was accelerating or decelerating. Thus the constant inlet velocity cannot indicate the swimming speed, and the fishes can not maintain formation configurations .

Because the net force of the fish was influenced by the St number, we conducted a series of simulations at different $S t$ to find the value that can ensure the net force to be zero. Firstly, a single fish 
was considered. The $S t$ number is tested from 0.35 to 0.6 shown in Table 1 . We find that the net force of the fish body is zero when the $S t$ number is 0.45 as shown in Figure 6.

Table 1. The St number in the single fish test.

\begin{tabular}{ccc}
\hline $\boldsymbol{R e}$ & Fluid Velocity & St \\
\hline 1000 & $1 L / s$ & $0.35,0.4,0.45,0.5,0.55,0.6$ \\
\hline
\end{tabular}

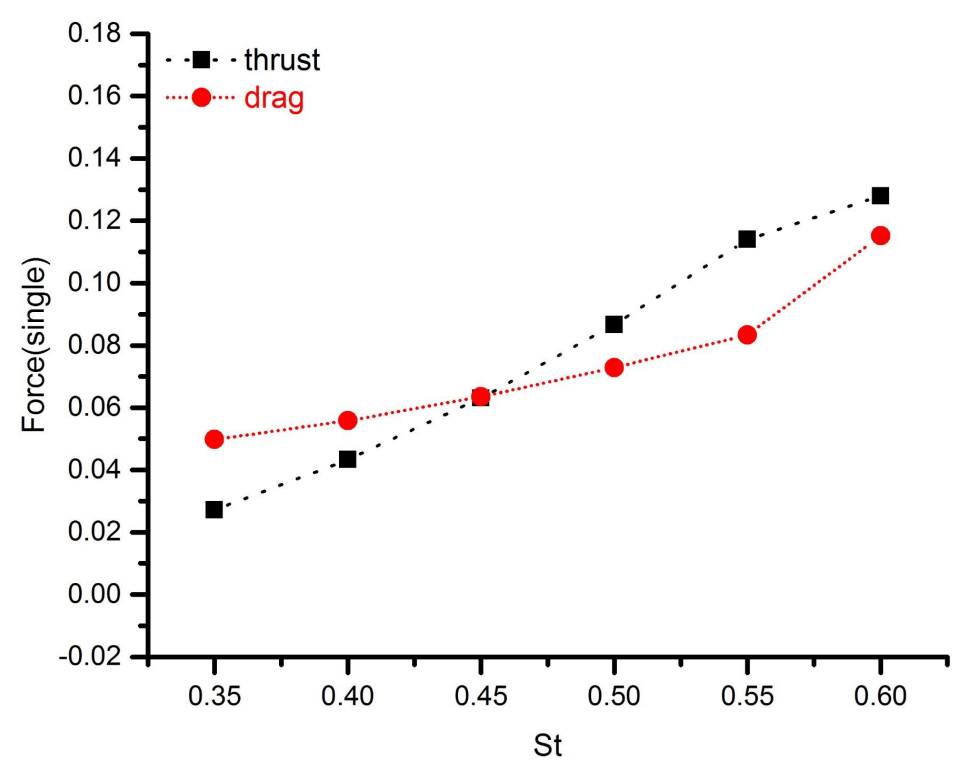

Figure 6. Results of simulations at different $S t$ : a single fish.

Then, the diamond swarm and the rectangle swarm were considered. The St number was tested from 0.15 to 0.625 , and the spacing between fishes is changed from $0.5 L$ to $2 L$ shown in Table 2 . We calculated the thrust force and drag force of each fish and the average net force, and a part of the results are shown in Figure 7.

Table 2. The St number and spacing in the diamond or rectangle swarm test.

\begin{tabular}{cccc}
\hline Re & Fluid Velocity & St & Spacing \\
\hline 1000 & $1 L / s$ & $0.15,0.3,0.4,0.45,0.5,0.625$ & $0.5 L, 0.75 L, 1.0 L, 1.25 L, 1.5 L, 1.75 L, 2.0 L$ \\
\hline
\end{tabular}
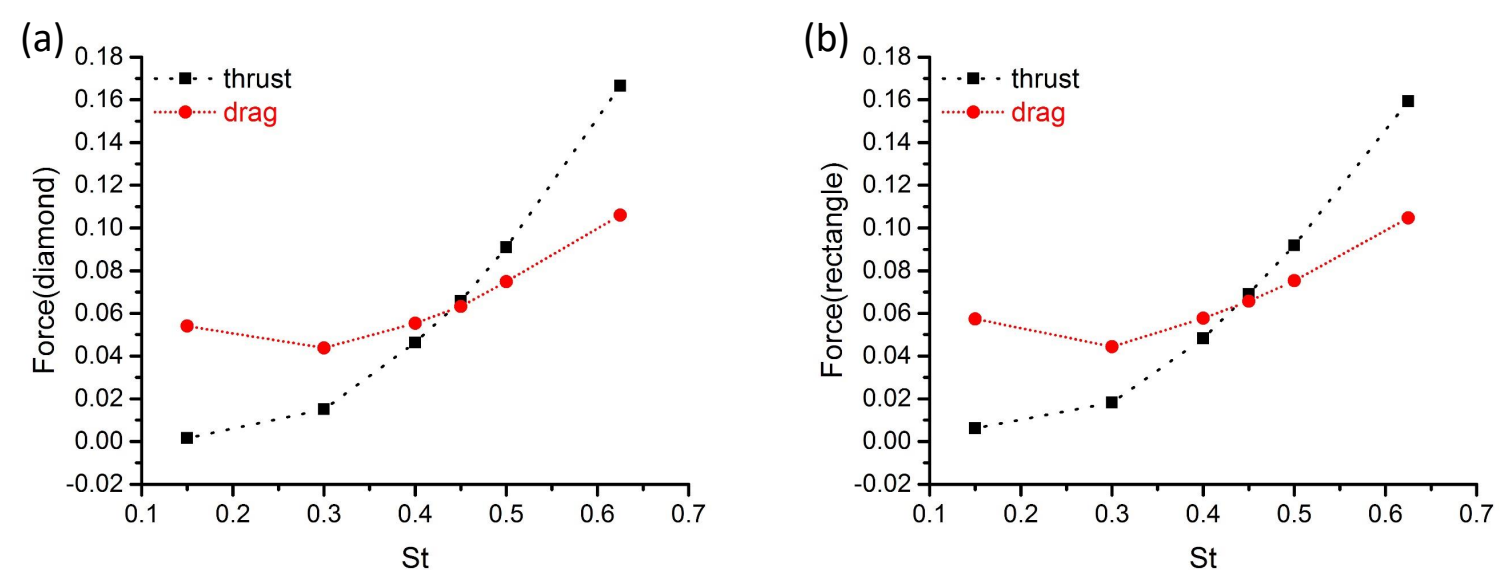

Figure 7. Results of the average force when the spacing is $1 L$ : (a) the diamond swarm, (b) the rectangle swarm. 
The results show that the average net force of the fish swarm was zero when the $S t$ number was near to 0.45 . Therefore, we also show the thrust and drag of each fish at each spacing under $S t=0.45$ in Figure 8. In this work, we think the net force was 0 when the difference between thrust and drag was less than $10 \%$. We also find that as the St number increased, the thrust also increased. This is because we changed the $S t$ number by setting different tail-beat frequency $f$. The larger tail-beat frequency $f$, the larger $S t$ number, and thrust. We set the $S t$ number to 0.45 in the subsequent simulation.

To simulate the swarm of fish-like robots, we mainly investigated four typical kinds of swarm formations, which are tandem, phalanx, diamond and rectangle. The tandem and phalanx swarm are shaped by three fishes. The diamond and rectangle swarm were shaped by four fishes. For each swarm shape, we changed the spacing between fishes and analyzed its effects on the fish swimming efficiency. The average performances of four swarm shapes were also compared. The experimental parameters are shown in Table 3.

(a)
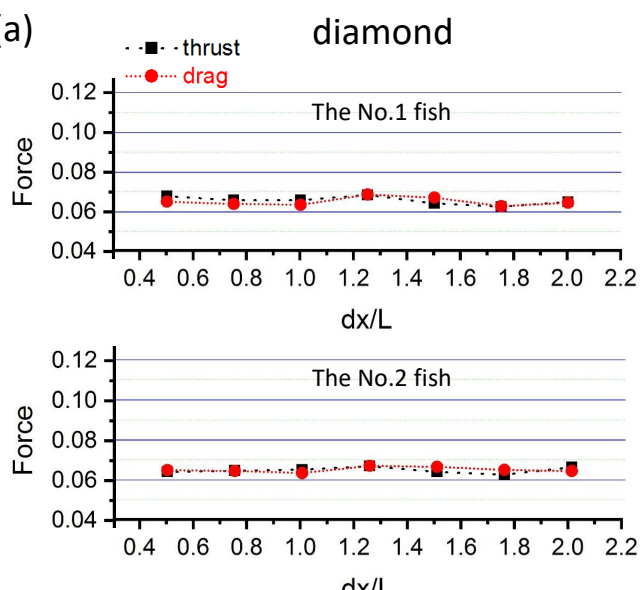

$\mathrm{dx} / \mathrm{L}$
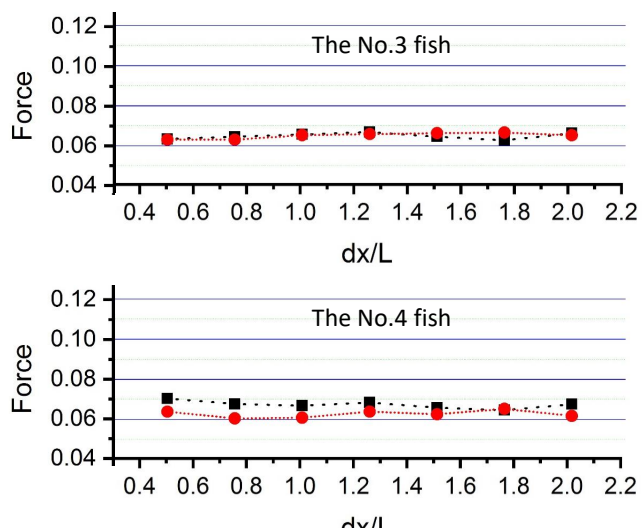

$\mathrm{dx} / \mathrm{L}$

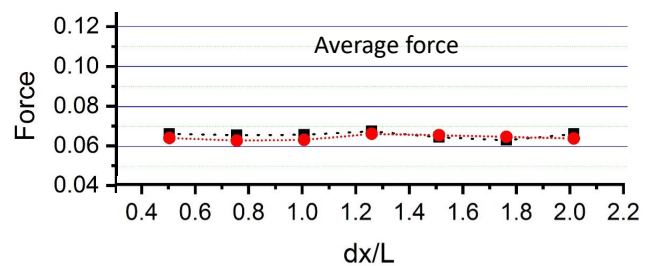

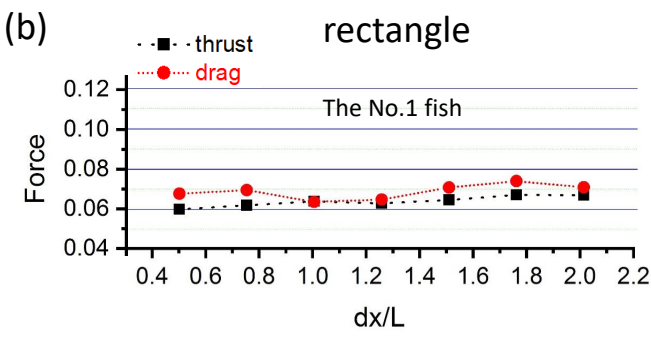

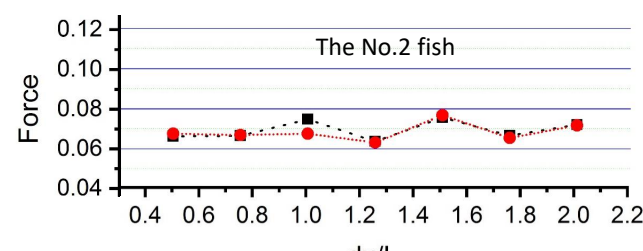

$\mathrm{dx} / \mathrm{L}$

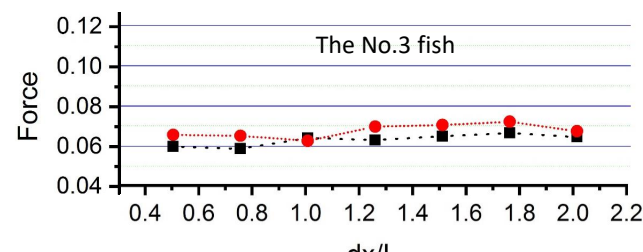

$\mathrm{dx} / \mathrm{L}$
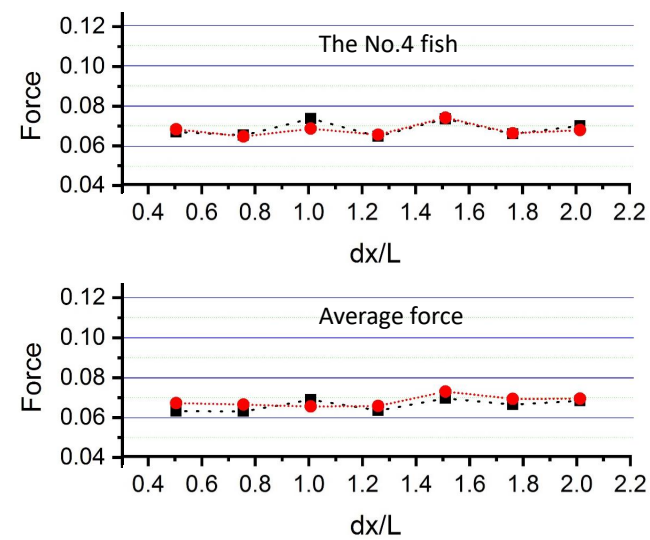

Figure 8. Results of the thrust and drag of each fish at each spacing: (a) the diamond swarm, (b) the rectangle swarm.

Table 3. The experimental parameters.

\begin{tabular}{ccccc}
\hline Re & Fluid Velocity & St & Swarm Shape & Spacing \\
\hline 1000 & $1 \mathrm{~L} / \mathrm{s}$ & 0.45 & tandem, phalanx, diamond, rectangle & $0.5 \mathrm{~L}, 0.75 \mathrm{~L}, 1.0 \mathrm{~L}, 1.25 \mathrm{~L}, 1.5 \mathrm{~L}, 1.75 \mathrm{~L}, 2.0 \mathrm{~L}$ \\
\hline
\end{tabular}




\subsection{The Tandem Swarm}

The thrust and drag at different spacing in the tandem swarm are shown in Figure 9. It is verified by simulations that the net force of fish is about zero. Therefore, the fishes are able to maintain the tandem formation, and the Froude efficiency $\eta$ is useful.

We calculated the non-dimensional thrust, lateral power loss coefficients and the Froude efficiency of each fish. The results are shown in Figure 10. In the tandem swarm, the performance of no. 2 and no. 3 fish was basically similar. In general, no. 2 fish has larger thrust coefficient, less lateral power loss and higher efficiency. However, as the spacing changes, we find that the performance of the fish is not always good. We illustrate this by analyzing the flow field in Figure 11 and Table 4.
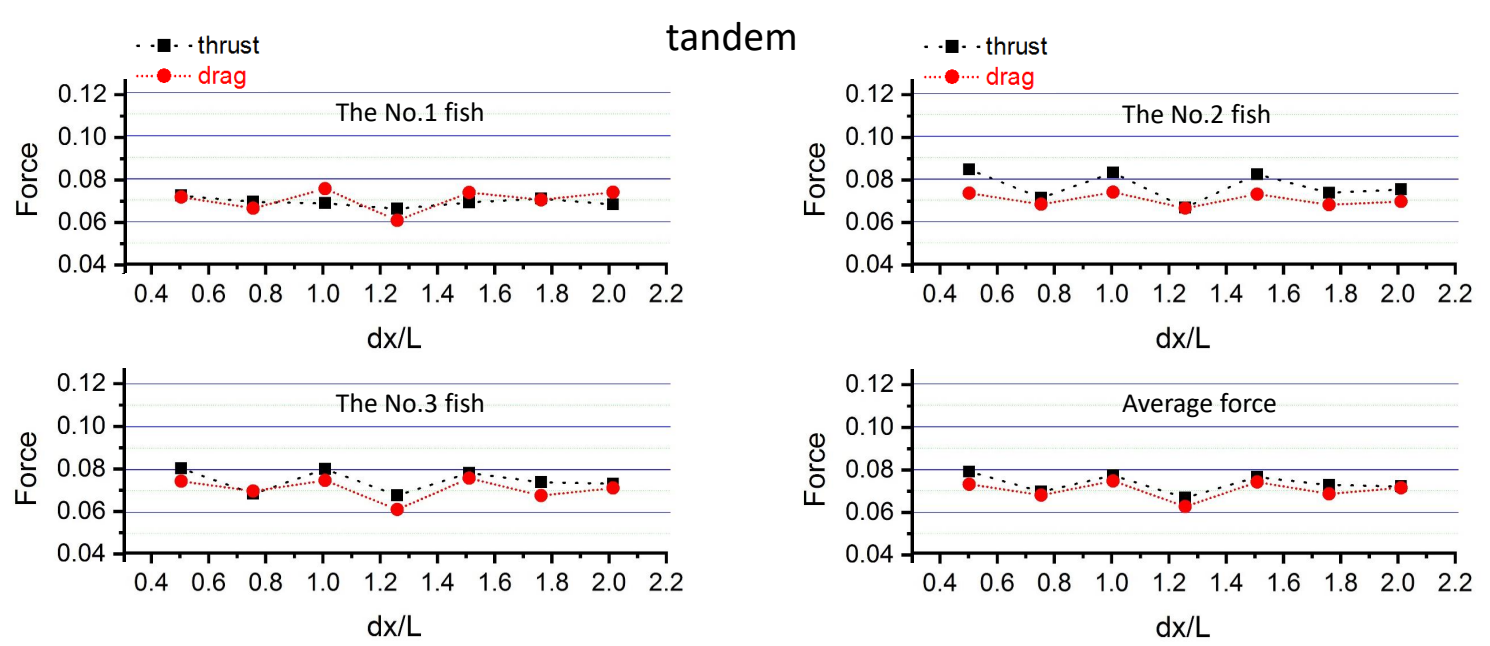

Figure 9. The thrust and drag at different spacing of the tandem swarm.

Near the head of the fish robot, if the velocity direction of fluid in vertical direction was the same as the direction of fish swinging, the fish had better efficiency [25]. In this work, the wake indicates the vertical velocity of fluid. The color of the wake indicates the value and direction of the vertical velocity. The velocity boundary is from $-0.25 \mathrm{~L} / \mathrm{s}$ to $0.25 \mathrm{~L} / \mathrm{s}$, where the blue wake indicates that the vertical direction fluids velocity is negative, and the red wake indicates that the vertical direction fluids velocity is positive. The deeper the color of the wake, the greater the relative velocity.

A wake flow had a diameter of approximately $0.24 \mathrm{~L}$ when it had just fallen off from the fish tail. The farther from the tail, the wake was weaker and the spacing between two wakes were larger, as shown in Figure 11a.

The no.2 fish achieved the best performance in the Froude efficiency. The Froude efficiency was the highest when the spacing between fishes was $1.0 \mathrm{~L}$, and it was the lowest when the spacing was $1.25 \mathrm{~L}$. This is determined by the wake of the no. 1 fish, the phase of the no. 2 fish and the spacing between fishes.

We measured the size of the blue wake and the red wake near the no. 2 fish head when the wake had just fallen off from the tail of the no.1 fish. When the spacing is $1.0 \mathrm{~L}$ and $1.25 \mathrm{~L}$, near the head of no. 2 fish, the sizes of the wake were basically equal. However, when the fish head swings downwards, the wake at the spacing of $1.0 \mathrm{~L}$ shown in Figure $11 \mathrm{~b}$ is the same direction as the fish swings. The wake at the spacing of $1.25 \mathrm{~L}$ shown in Figure $11 \mathrm{~d}$ is opposite to the direction of the fish swings. Similarly, when the fish head swings upwards, the wake is the same direction as the fish swings at the spacing of $1.0 \mathrm{~L}$ shown in Figure 11c. The wake is opposite to the direction of the swinging of fish at the spacing of $1.25 \mathrm{~L}$ shown in Figure 11e. The wake directions and sizes of other spacings are shown in Table 4 . It is only necessary to discuss the wake in which the fish head swings downwards, because that of the upward swing is similar. 
(a)

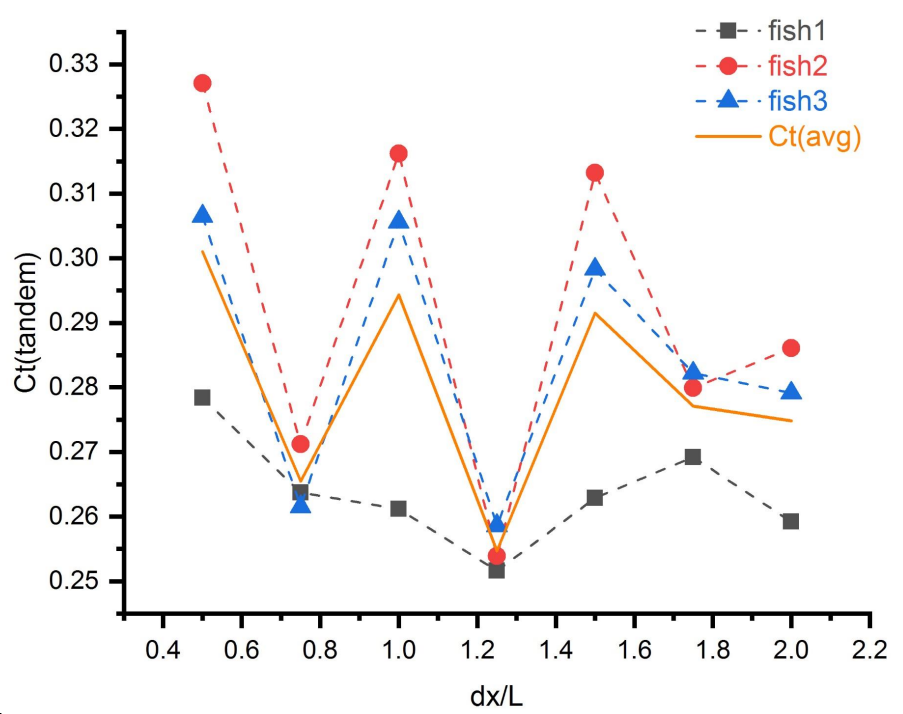

(b)

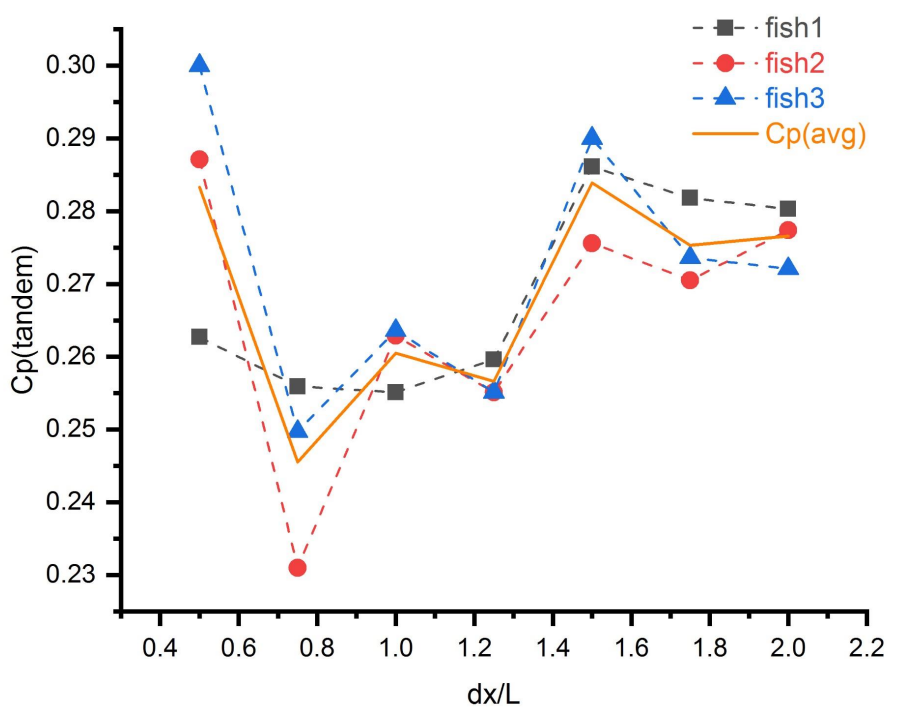

(c)

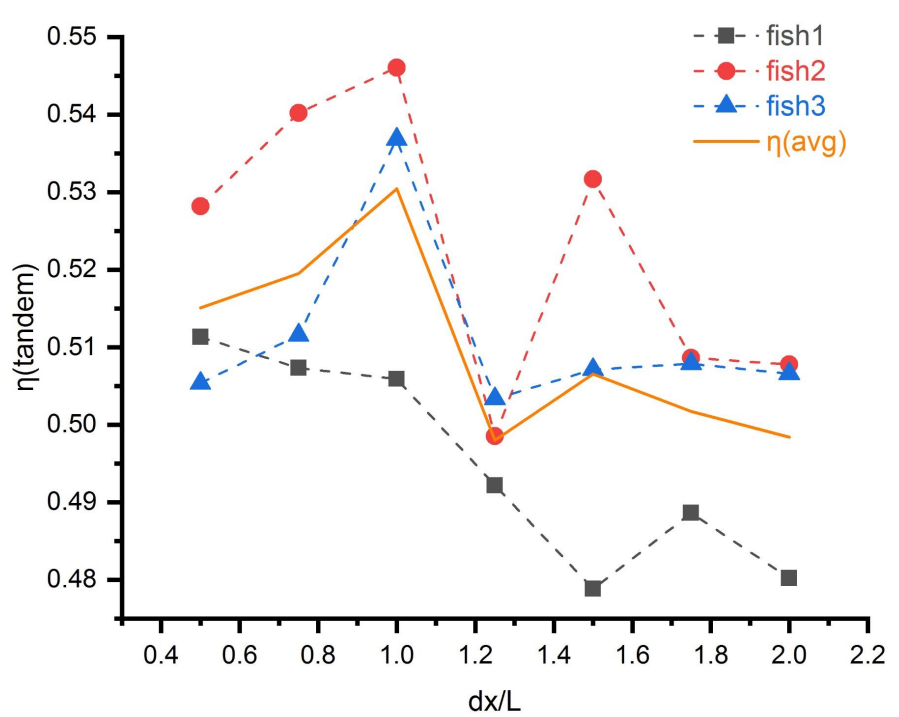

Figure 10. Results of the tandem swarm: (a) non-dimensional thrust coefficients $C_{t}$, (b) non-dimensional lateral power loss coefficients $C_{p}$, (c) the Froude efficiency $\eta$. 

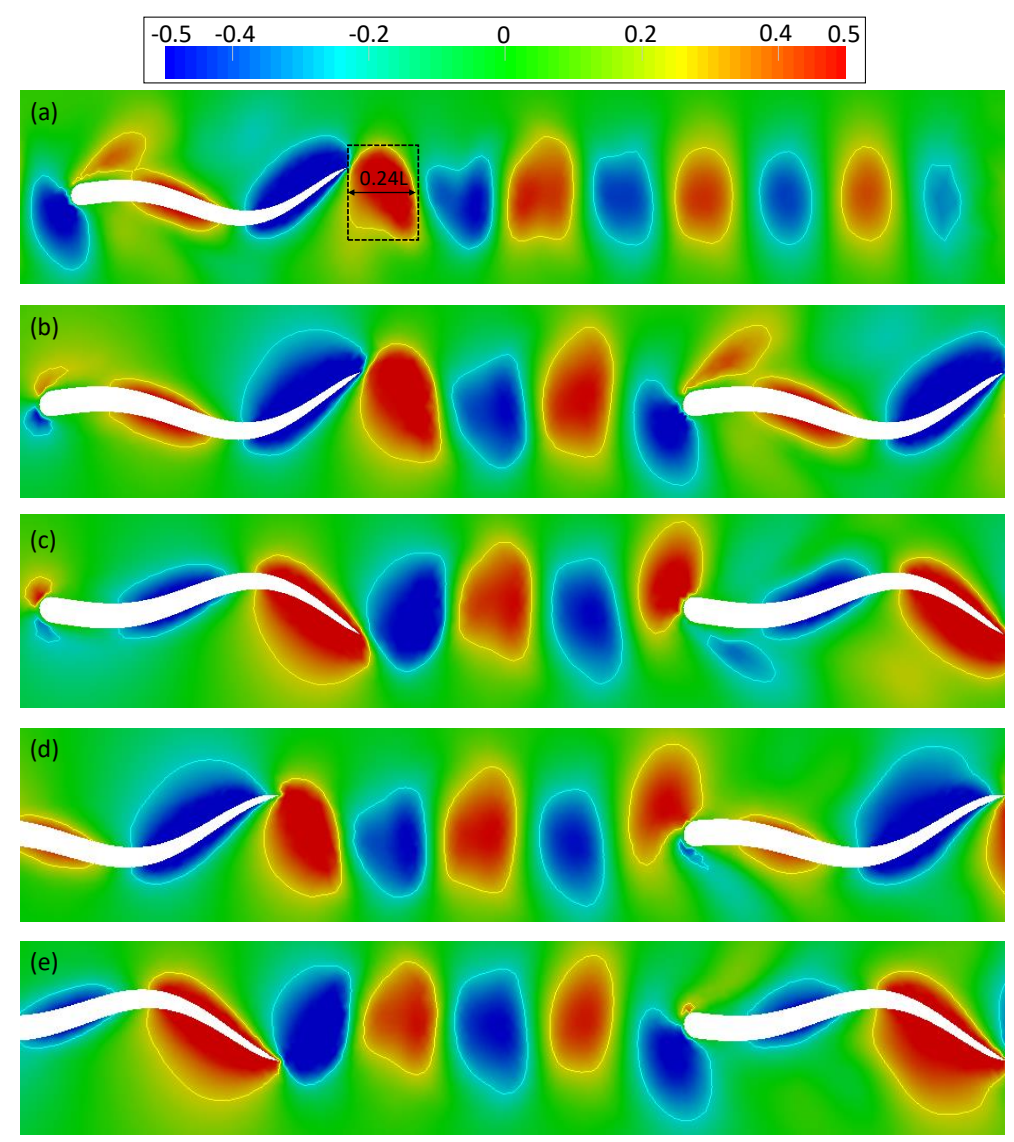

Figure 11. The wake of the tandem swarm: (a) the size and location of the wake; at the spacing of 1.0L: (b) the fish head swings downwards, (c) the fish head swings upwards; at the spacing of 1.25L: (d) the fish head swings downwards, (e) the fish head swings upwards.

Table 4. The wake directions and sizes near the head of no.2 fish.

\begin{tabular}{ccc}
\hline Spacing & The Wake Direction & The Wake Size \\
\hline $0.5 L$ & up & $0.023 L$ \\
$0.75 L$ & down & $0.027 L$ \\
$1.0 L$ & down & $0.144 L$ \\
$1.25 L$ & up & $0.118 L$ \\
$1.5 L$ & down & $0.079 L$ \\
$1.75 L$ & up & $0.039 L$ \\
$2.0 L$ & down & $0.053 L$ \\
\hline
\end{tabular}

When the spacing was $0.5 \mathrm{~L}$, the wake in the opposite direction did not result in very low efficiency because the size of the wake is small. The wake became weaker as the spacing increased. Therefore, at large spacing such as $1.75 \mathrm{~L}$ and $2.0 \mathrm{~L}$, whether there was a positive or negative wake, it had little effect on efficiency. The efficiency of the single fish was about 0.47 which was lower than the minimum efficiency of no. 2 fish. It shows that in a stable formation of fishes at a constant spacing and speed, even the effect of the worst wake was better than that of no wake.

Therefore, we conclude that the wake is consistent with the direction of the fish head, which can improve swimming efficiency. In addition, the large and strong wake influences more on the Froude efficiency. Also, the pressure generated by the swinging of the fish body can hardly affect the adjacent fish in the tandem swarm. 


\subsection{The Phalanx Swarm}

The experiments find that when the spacing between fishes was $0.5 \mathrm{~L}$, the net force of the fish body was not zero. The results are shown in Figure 12. Through Ashraf's experiment [47] and Dai's simulation [29], we learn that in the high-efficiency phalanx fish swarm at the spacing between $0.15 \mathrm{~L}$ and $0.65 L$, fishes swim by "out-phase". However, in this work, all fish swim by "in-phase". Therefore, the drag of the phalanx swarm at close spacing $(0.5 \mathrm{~L})$ was larger than thrust. Thus, in this work, the phalanx swarm does not discuss the case where the spacing between fishes is $0.5 \mathrm{~L}$.
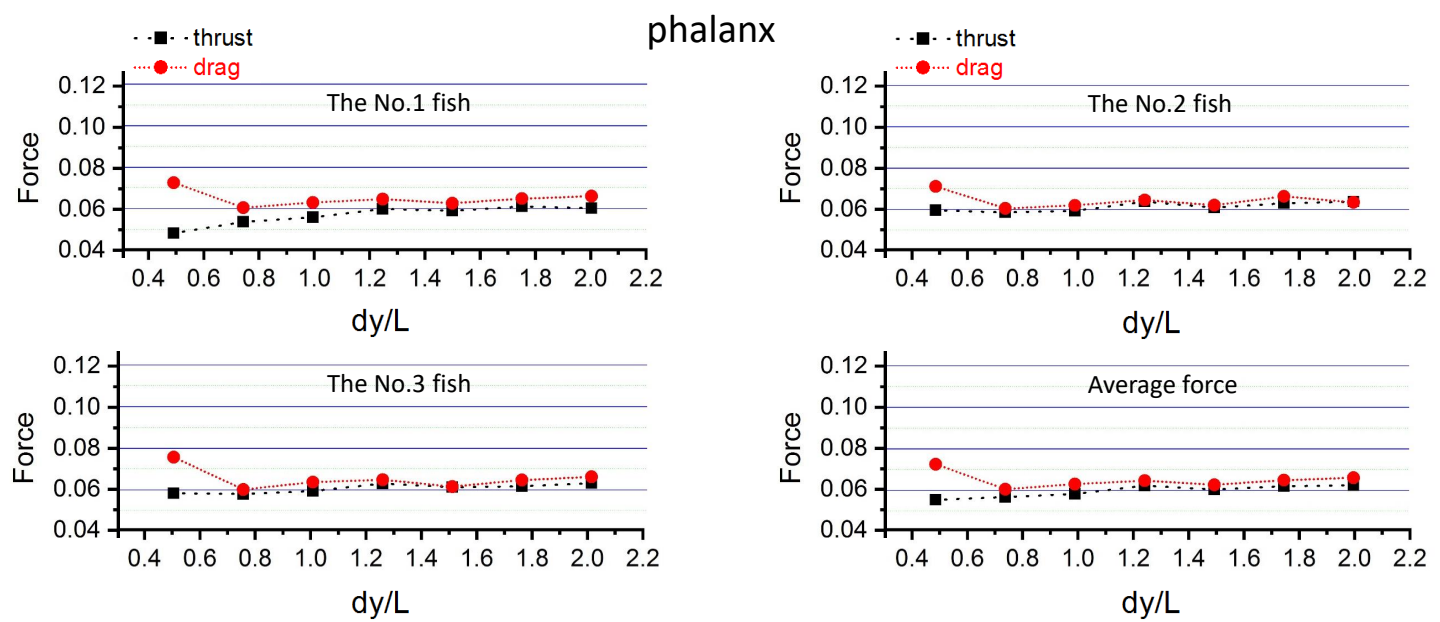

Figure 12. The thrust and drag at different spacing of the phalanx swarm.

We calculate the non-dimensional thrust, lateral power loss coefficients and the Froude efficiency of each fish in other spacing cases. The results are shown in Figure 13. In the phalanx swarm, the thrust coefficient of the three fishes had the same trend as the spacing changes, and the values of fishes on both sides were close. The no. 2 fish had the smallest lateral power loss. However, there was no fish which had the best Froude efficiency.

From the phalanx swarm, we can get that the pressure generated by the swinging of fish body will affect the adjacent fish. When the spacing between fishes was $0.75 \mathrm{~L}$, the contours of the pressure field is shown in Figure 14a. We find that the pressure fields of adjacent fishes overlap and the Froude efficiency was the lowest. Figure 14b shows the contours of pressure field when the spacing was $1.25 \mathrm{~L}$. The pressure fields of each fish are independent, and do not affect each other and the Froude efficiency is better. In a word, if the spacing between fishes was too close, the fish swam unsteadily and reduced the swimming efficiency. 
(a)

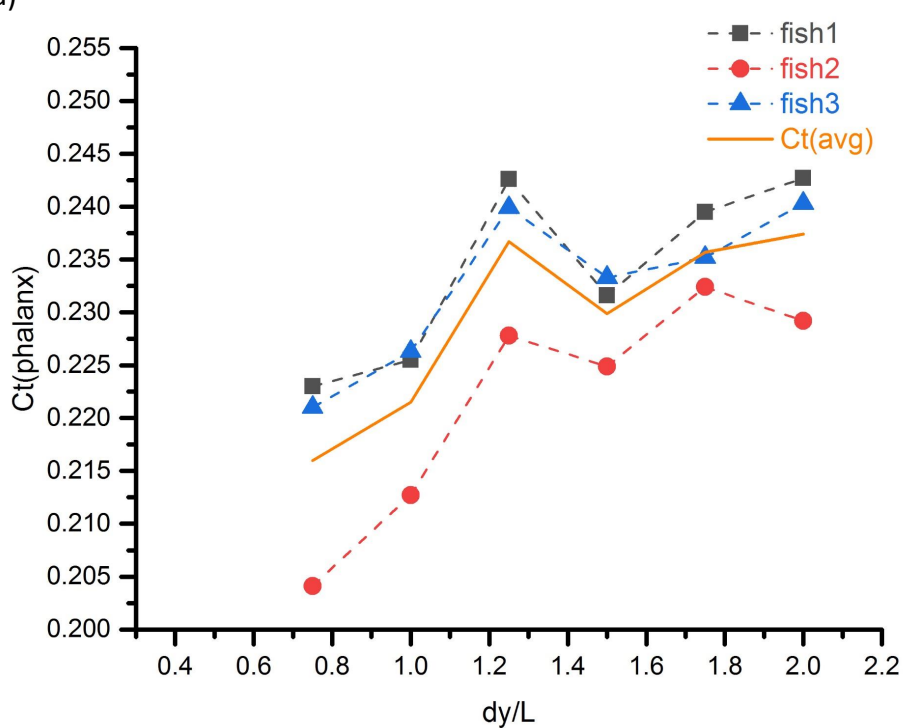

(b)

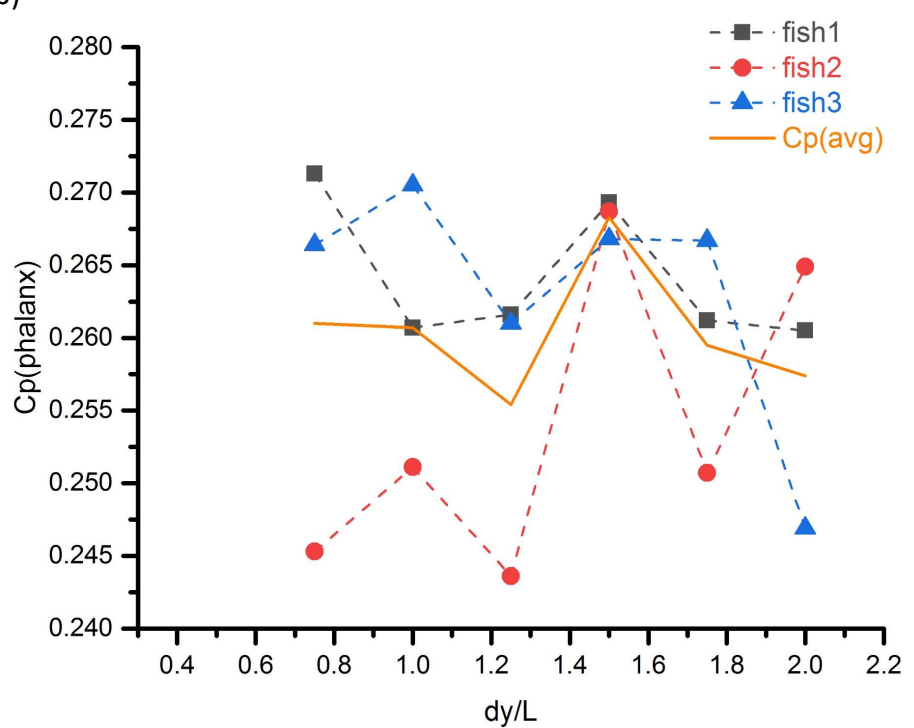

(c)

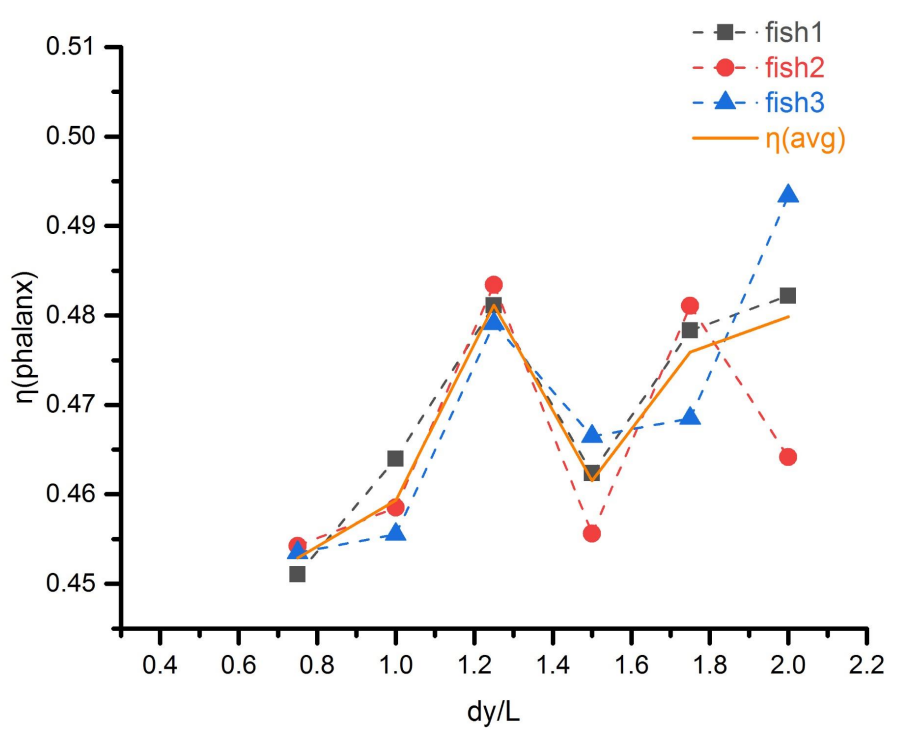

Figure 13. Results of the phalanx swarm: (a) non-dimensional thrust coefficients $C_{t}$, (b) non-dimensional lateral power loss coefficients $C_{p}$, (c) the Froude efficiency $\eta$. 


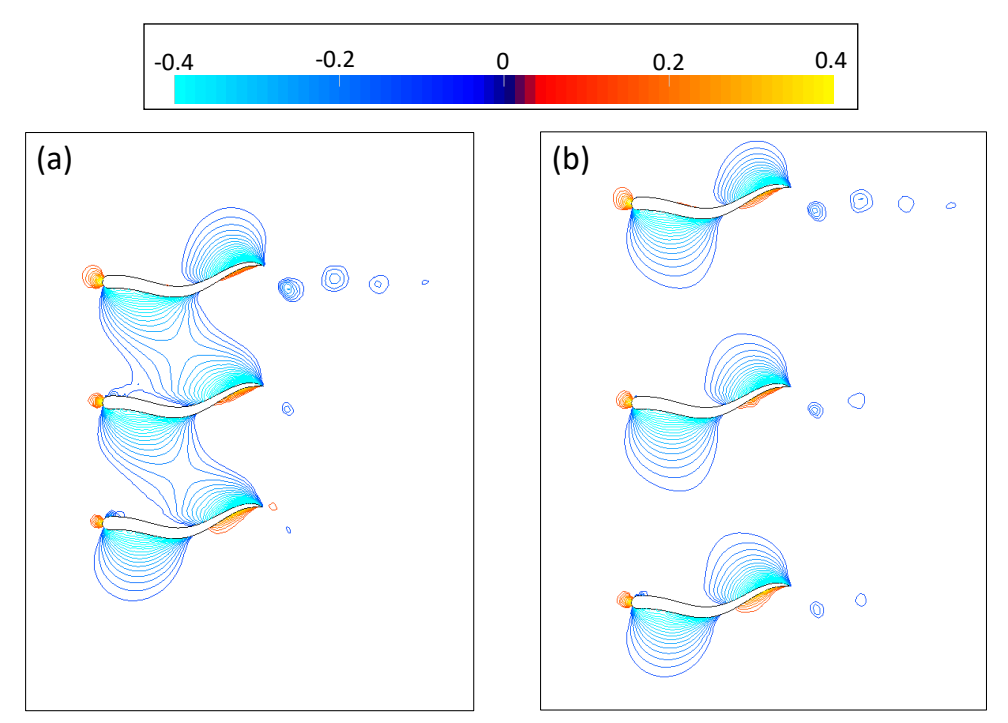

Figure 14. The contours in pressure field of the phalanx swarm: (a) spacing $=0.75 \mathrm{~L},(\mathbf{b})$ spacing $=1.25 \mathrm{~L}$.

\subsection{The Diamond Swarm}

The thrust and drag at different spacing in the diamond swarm are shown in Figure 8a. It is verified by simulations that the net force of each fish is about zero. Thus, the fishes are able to maintain the diamond formation, and the Froude efficiency $\eta$ is useful.

We calculated the non-dimensional thrust, lateral power loss coefficients and the Froude efficiency of each fish. The results are shownin Figure 15. In the diamond swarm, the thrust coefficients of no. 2 fish and no. 3 fish in the symmetrical position were basically equal, and the No.4 fish had the largest thrust coefficient. None of the fish had an obvious advantage in the lateral power loss and the Froude efficiency. The no.2 fish and the no.3 fish basically had the same results after the spacing was larger than $1.0 \mathrm{~L}$.

In the diamond swarm, only the no.4 fish was affected by the wake of the fish in front. When the spacing was large, there was almost no wake near the no.4 fish head as shown in Figure 16a, so the efficiency was not high. However, at close spacing shown as Figure 17a, the pressure generated by the adjacent fish swing affected the stability of the fish and reduced efficiency. Therefore, in the diamond swarm, the efficiency was best when the spacing was moderate $(1.25 \mathrm{~L})$. Its wake and pressure field are shown in Figures $16 \mathrm{~b}$ and $17 \mathrm{~b}$. The wake was weak, but it was better than no wake. 
(a)

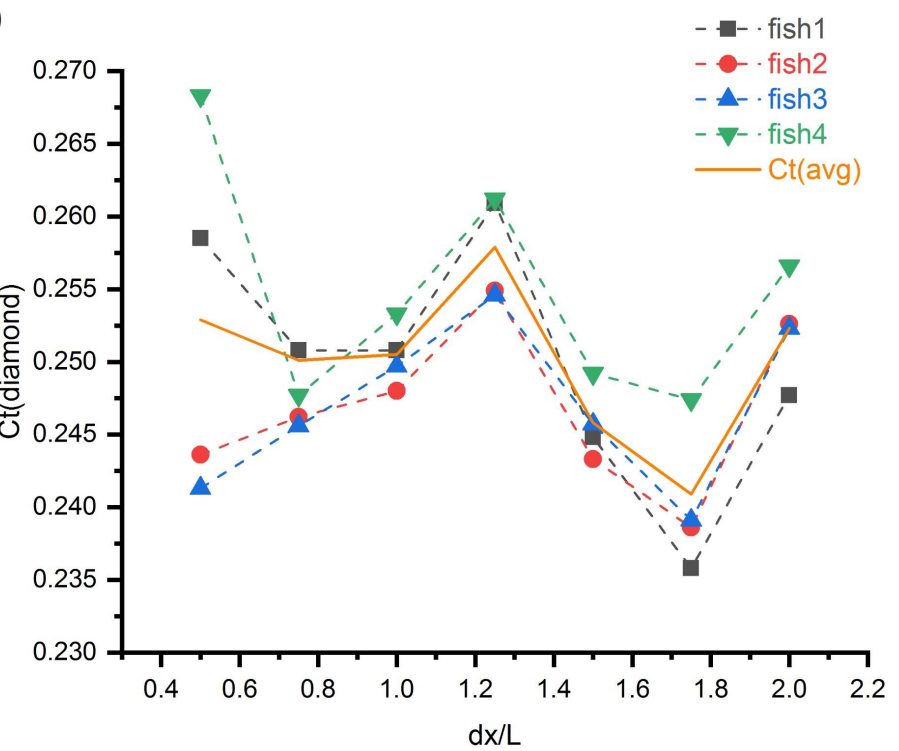

(b)

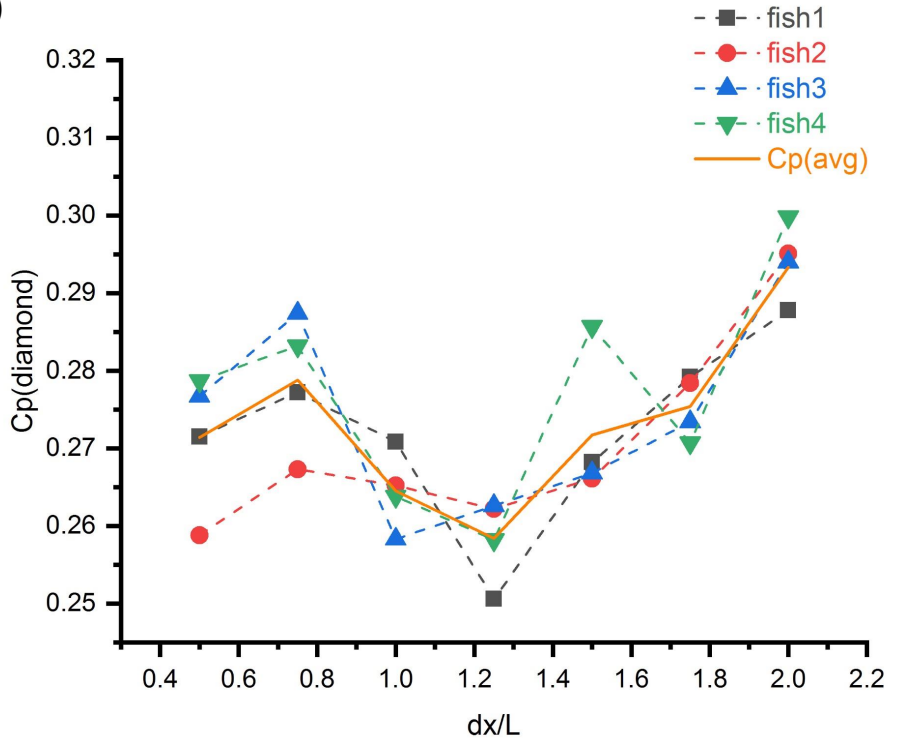

(c)

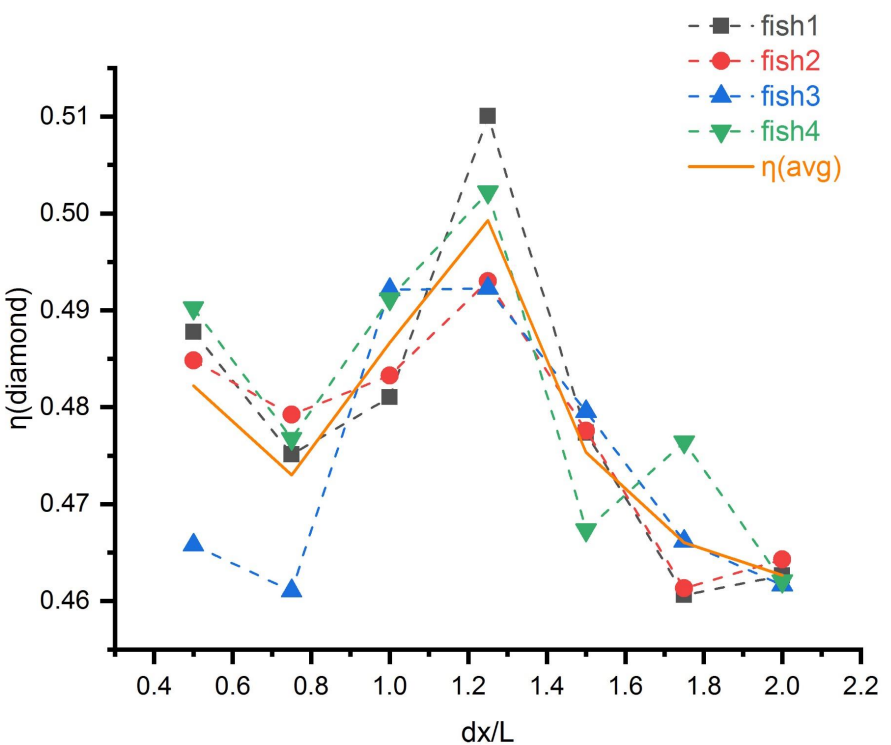

Figure 15. Results of the diamond swarm: (a) non-dimensional thrust coefficients $C_{t}$, (b) non-dimensional lateral power loss coefficients $C_{p},(\mathbf{c})$ the Froude efficiency $\eta$. 

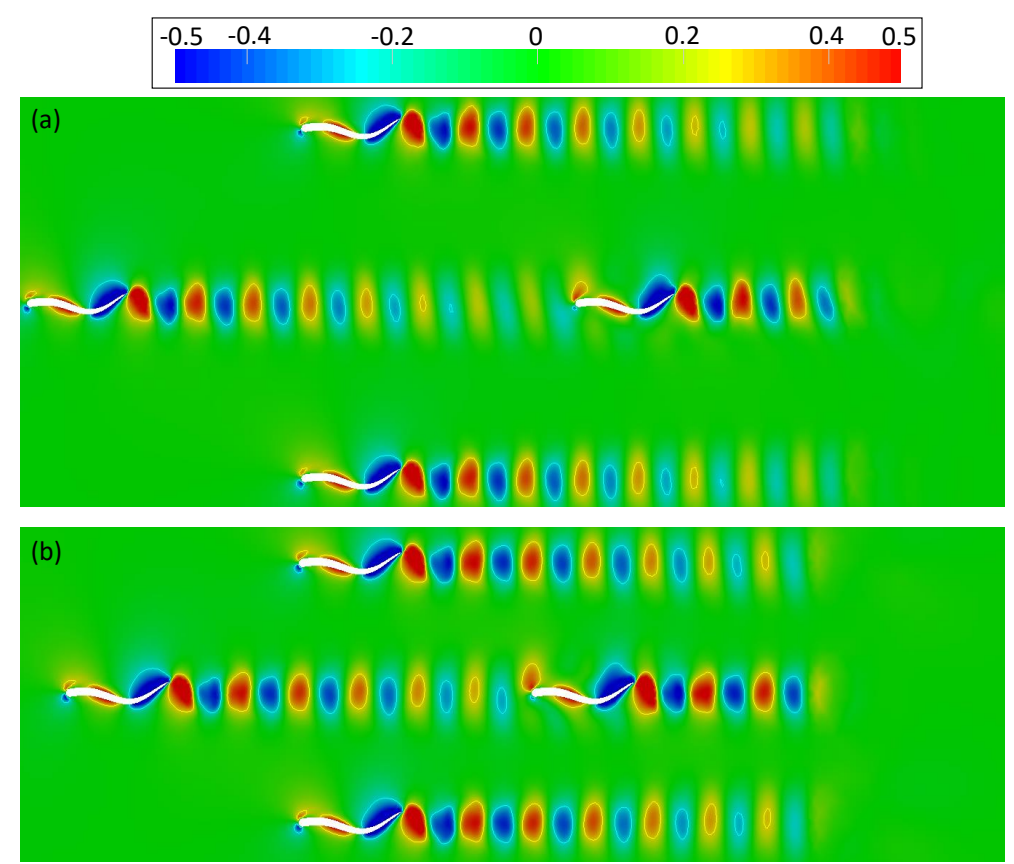

Figure 16. The wake of the diamond swarm: (a) spacing $=1.75 \mathrm{~L},(\mathbf{b})$ spacing $=1.25 \mathrm{~L}$.

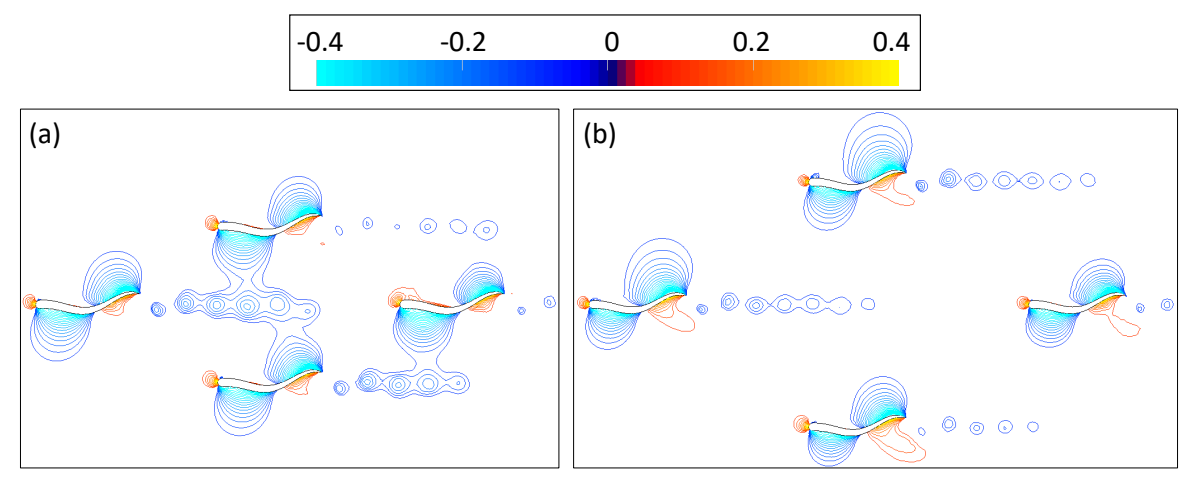

Figure 17. The contours in pressure field of the diamond swarm: (a) spacing $=0.75 \mathrm{~L}$, (b) spacing $=1.25 \mathrm{~L}$.

\subsection{The Rectangle Swarm}

The thrust and drag at different spacing in the rectangle swarm are shown in Figure $8 \mathrm{~b}$. It is verified by simulations that the net force of each fish is about zero. Therefore, the fishes were able to maintain the rectangle formation, and the Froude efficiency $\eta$ is useful.

We calculated the non-dimensional thrust, lateral power loss coefficients and the Froude efficiency of each fish. The results are shown in Figure 18. In the rectangle swarm, the thrust coefficients of no.1 fish and no. 3 fish, no.2 fish and no. 4 fish in the symmetrical position were basically equal. None of the fish had an obvious advantage for the lateral power loss and the Froude efficiency. Nevertheless, the performance of fish behind was better than that of fish in front.

In the rectangle swarm, at close spacing, the fish was affected by the pressure of the adjacent fish swing and resulting in low efficiency; at large spacing, the wake was small and weak, which also leads to low efficiency. Therefore, the moderate spacing $(1.5 \mathrm{~L})$ made the rectangle swarm the most efficient. The wake is shown in Figure 19, and the pressure field is shown in Figure 20. 
(a)

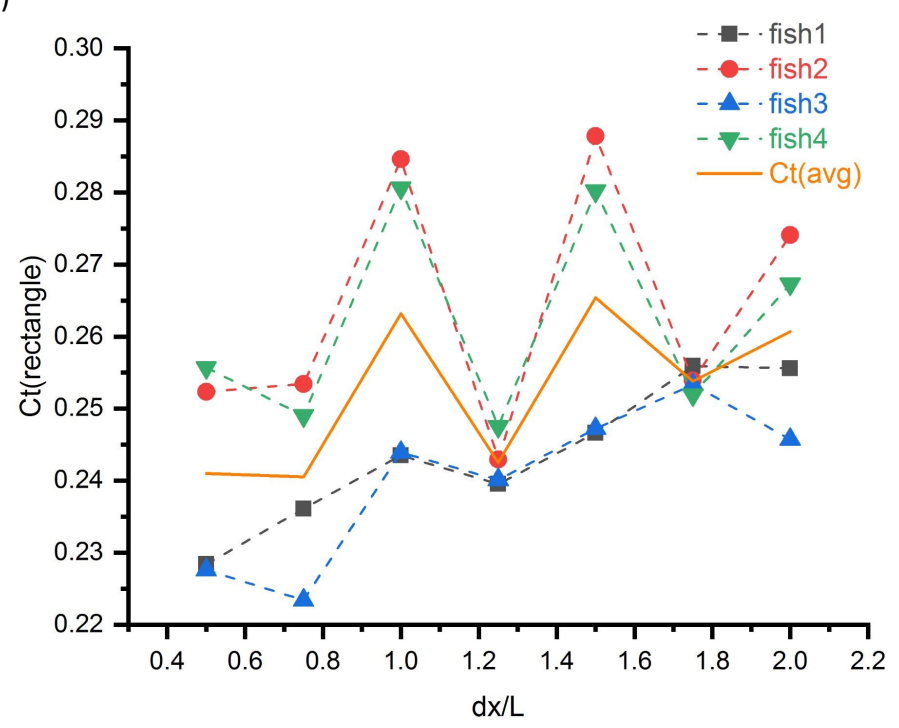

(b)

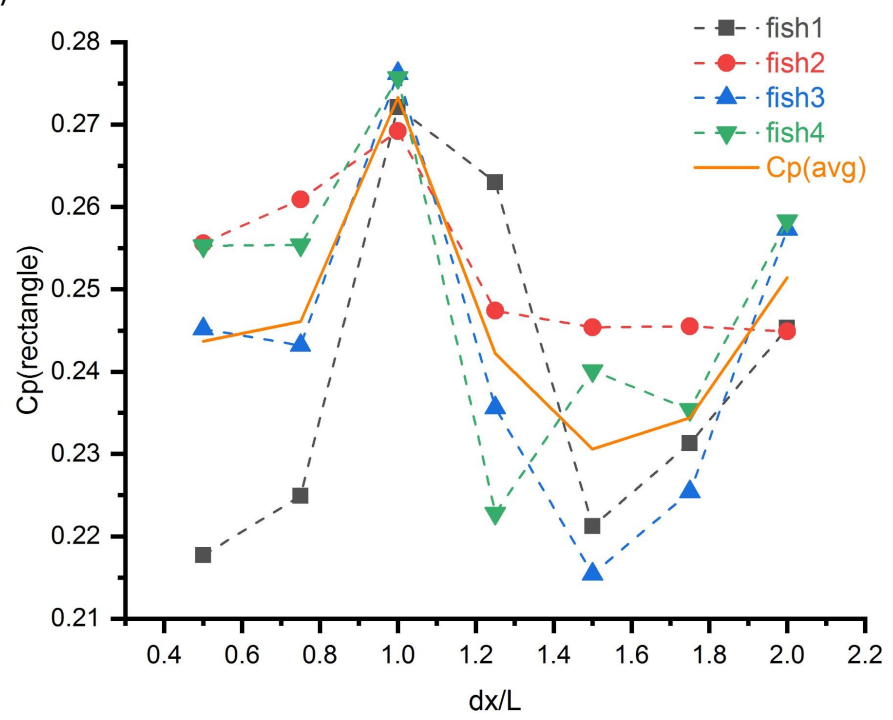

(c)

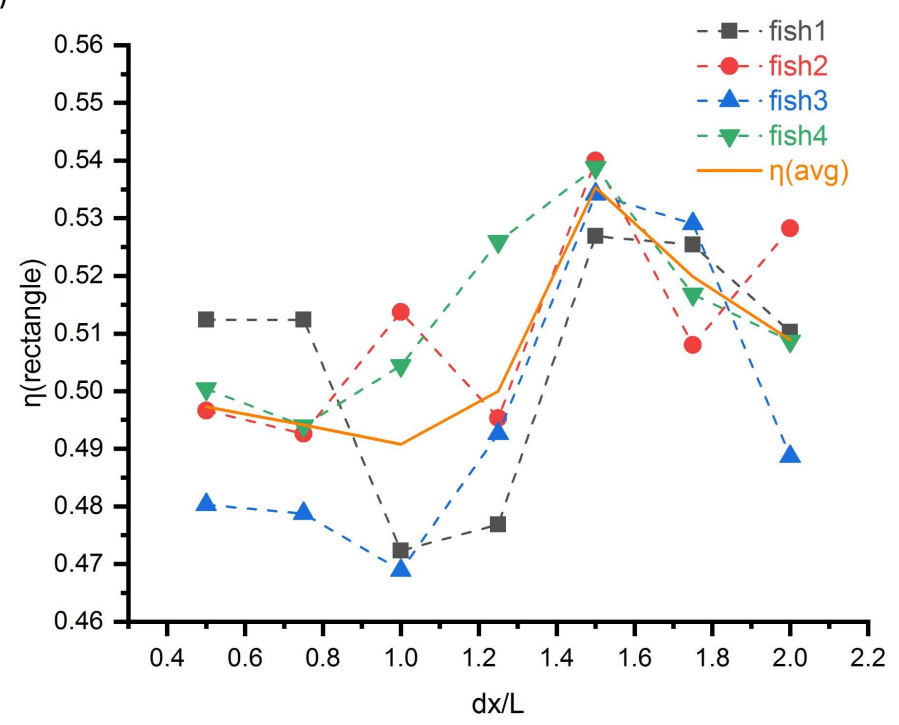

Figure 18. Results of the rectangle swarm: (a) non-dimensional thrust coefficients $C_{t},(\mathbf{b})$ non-dimensional lateral power loss coefficients $C_{p}$, (c) the Froude efficiency $\eta$. 


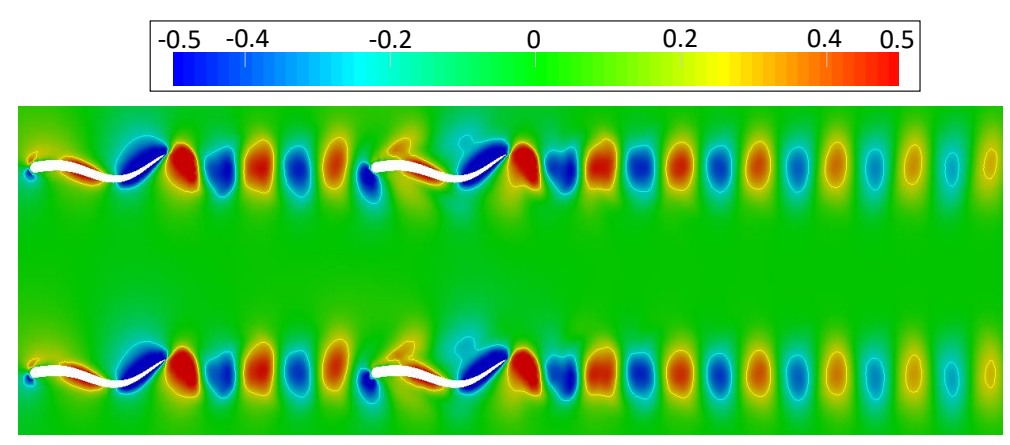

Figure 19. The wake of the rectangle swarm at the spacing of $1.5 \mathrm{~L}$.

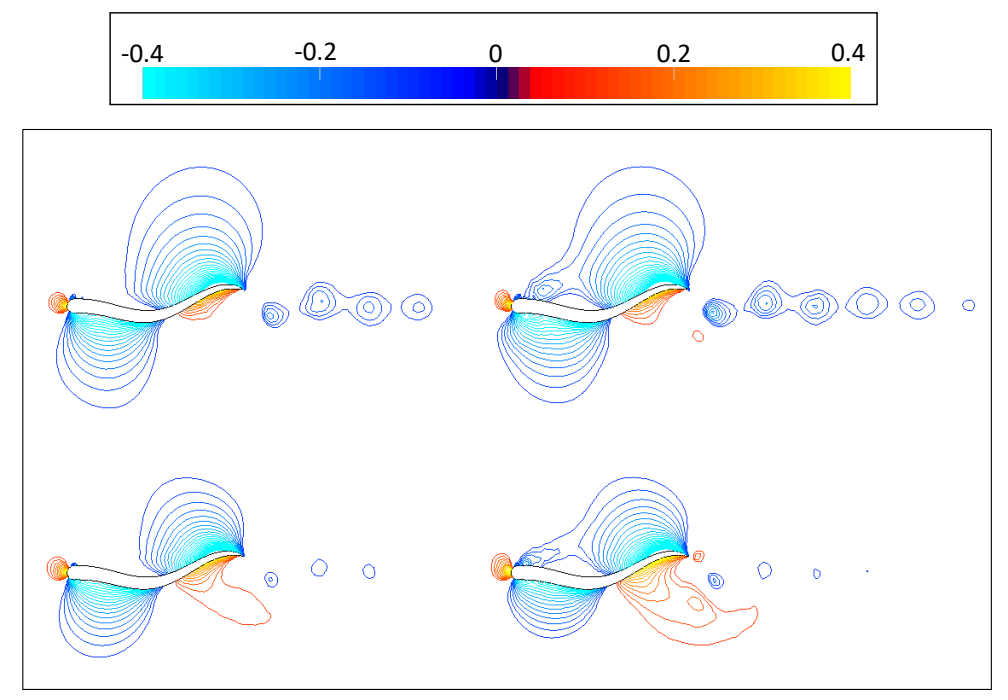

Figure 20. The contours in pressure field of the rectangle swarm at the spacing of $1.5 \mathrm{~L}$.

\subsection{Comparison between Different Shapes}

We only compared the average Froude efficiency of the different fish-like robots swarms. The performance of a single fish-like robot was also simulated as a baseline. As shown in Figure 21, in the stable formation configurations of fishes at a constant spacing and speed, the tandem swarm has the highest efficiency when the spacing is less than $1.25 \mathrm{~L}$, while the rectangle swarm was more efficient when the spacing was larger than $1.25 \mathrm{~L}$. As the spacing increased, the efficiency of the diamond swarm was lower than that of a single fish, and the performance of the phalanx swarm was the worst.

There were two factors that affected the Froude efficiency: thrust and lateral power loss. The thrust was large at close spacing, but the side-by-side fish caused a large lateral power loss, so the tandem swarm has the highest Froude efficient at close spacing. At large spacing, the rectangle swarm and the diamond swarm were almost unaffected by side-by-side fish. However, the rectangle swarm can obtain stronger and larger wakes than the diamond swarm, so the efficiency of the rectangle swarm was the highest. The phalanx swarm had the worst performance because it was not affected by the wake.

The simulation results suggest that we should choose the tandem swarm when the spacing between fishes is less than $1.25 \mathrm{~L}$, and should choose the rectangle swarm when the spacing is larger. 


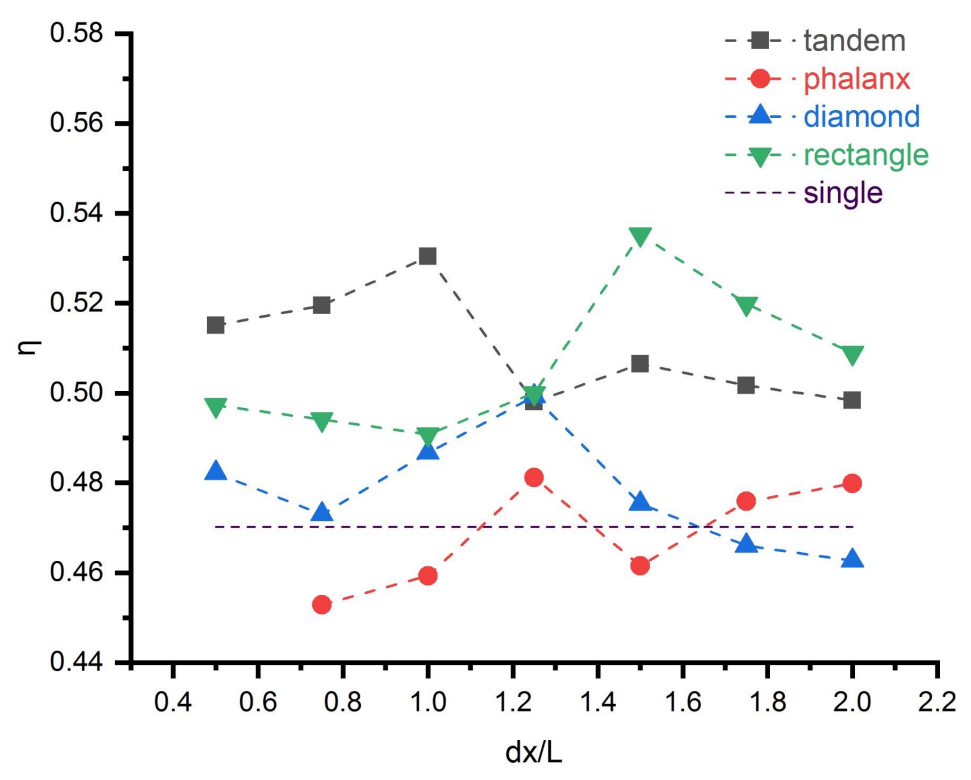

Figure 21. The average Froude efficiency.

\section{Conclusions}

In this work, we present the numerical simulation results of fish-like robots by changing the swarm configuration. All our conclusions are based on the stable formation of fishes at a constant spacing and speed. The results show that the wake and the pressure resulting from the swing of the fish body are important factors affecting the Froude efficiency. Precisely, the wake mainly affects the thrust, and the pressure resulting from the swing of the fish body mainly affects the lateral power loss. Generally, both the larger wake in the same direction as the swing of fish head and the less pressure resulting from the swing of the fish body lead to higher Froude efficiency. By comparing the performance of different fish swarms and analyzing the velocity fields and pressure fields, we can find that at close spacing the fish in the back can obtain large thrust coefficient because of the large wake from the front fish, but side-by-side fish can cause large lateral power loss. On the contrary, at large spacing, the fish obtain the small wake, which results in the small thrust coefficient, but the side-by-side fish hardly affect each other. By comparing the Froude efficiency between different swarms, the efficiency of the tandem swarm is highest at close spacing, and the efficiency of the rectangle swarm is highest at large spacing. The results suggest that the fish-like robot group could choose the suitable swarm configuration according to the different environment and tasks. In the future, we will further consider the three-dimensional model, and choose a larger Reynolds number, and simulate the turbulence flow. In addition, in the literature [29], we have learned that "out-phase" swimming seems to be more efficient than "in-phase" swimming, so changing the phase difference between adjacent fish-like robots will be a possible direction for future work. In order to make the simulation closer to reality, we will also consider more control algorithms.

Author Contributions: S.L. carried out the numerical simulations, data analysis and wrote the first draft of the manuscript. W.Y. and X.C. conceived and supervised the study and edited the manuscript. C.L. and L.X. contributed to the experiments design and the experimental platform. All authors reviewed the manuscript.

Funding: This research was funded by the National Natural Science Foundation of China (Grant No.91648204, Grant No.61802426), Science Challenge Project (No.TZ2017002, No.TZ2016002).

Conflicts of Interest: The authors declare no conflict of interest. 


\section{References}

1. Abukhalil, T.; Patil, M.; Sobh, T. A comprehensive survey on decentralized modular swarm robotic systems and deployment environments. Int. J. Eng. (IJE) 2013, 7, 44-60.

2. Caglioti, V.; Citterio, A.; Fossati, A. Cooperative, distributed localization in multi-robot systems: A minimum-entropy approach. In Proceedings of the IEEE Workshop on Distributed Intelligent Systems: Collective Intelligence and Its Applications, Prague, Czech Republic, 15-16 June 2006; pp. 25-30.

3. Fox, D.; Burgard, W.; Kruppa, H.; Thrun, S. A probabilistic approach to collaborative multi-robot localization. Auton. Robot. 2000, 8, 325-344. [CrossRef]

4. Baglietto, M.; Paolucci, M.; Scardovi, L.; Zoppoli, R. Information-based multi-agent exploration. In Proceedings of the Third International Workshop on Robot Motion and Control, Bukowy Dworek, Poland, 11 November 2002; pp. 173-179.

5. Treaty, N.A. Multi-Robot Systems in Military Domains; NATO Research and Technology Organisation: Neuilly-sur-Seine, France, 2008.

6. Stroupe, A.; Huntsberger, T.; Okon, A.; Aghazarian, H.; Robinson, M. Behavior-based multi-robot collaboration for autonomous construction tasks. In Proceedings of the 2005 IEEE/RSJ International Conference on Intelligent Robots and Systems (IROS 2005), Edmonton, AB, Canada, 2-6 August 2005; pp. 1495-1500.

7. Patil, M.D.; Abukhalil, T. Design and implementation of heterogeneous robot swarm. In Proceedings of the ASEE 2014 Zone I Conference, University of Bridgeport, Bridgpeort, CT, USA, 3-5 April 2014; pp. 3-5.

8. Patil, M.; Abukhalil, T.; Patel, S.; Sobh, T. UB robot swarm-Design, implementation, and power management. In Proceedings of the 2016 12th IEEE International Conference on Control and Automation (ICCA), Kathmandu, Nepal, 1-3 June 2016; pp. 577-582.

9. Patil, M.; Abukhalil, T.; Sobh, T. Hardware Architecture Review of Swarm Robotics System: Self-Reconfigurability, Self-Reassembly, and Self-Replication. ISRN Robot. 2013, 2013, 849606. [CrossRef]

10. Patil, M.; Abukhalil, T.; Patel, S.; Sobh, T. UB Swarm: Hardware implementation of heterogeneous swarm robot with fault detection and power management. Int. J. Comput. 2016, 15, 162-176.

11. Abukhalil, T.Y.; Patil, M.; Sobh, T.M. UBSwarm: Design of a Software Environment to Deploy Multiple Decentralized Robots. In Proceedings of the 2014 Zone 1 Conference of the American Society for Engineering Education, Bridgeport, CT, USA, 3-5 April 2014.

12. Balch, T.; Arkin, R.C. Behavior-based formation control for multirobot teams. IEEE Trans. Robot. Autom. 1998, 14, 926-939. [CrossRef]

13. Fax, J.A.; Murray, R.M. Information flow and cooperative control of vehicle formations. IEEE Trans. Autom. Control 2004, 49, 1465-1476. [CrossRef]

14. Ji, X.; Wu, S.; Liu, X.; Du, Y.; Tang, J. Research and design on physical multi-UAV system for verification of autonomous formation and cooperative guidance. In Proceedings of the 2010 International Conference on Electrical and Control Engineering (ICECE), Wuhan, China, 25-27 June 2010; pp. 1570-1576.

15. De Silva, D. Formation Control for Unmanned Aerial Vehicles. Master's thesis, Instituto Superior Tecnico (IST), Lisboa, Portugal, 2012.

16. Shree, A.S.; Singh, V.K.; Solanki, P.B.; Behera, L. Design and Development of Robotic Fish Swarm Based Coast Monitoring System. In Proceedings of Conference on Advances In Robotics, Pune, India, 4-6 July 2013; ACM: New York, NY, USA; pp. 1-6.

17. Takada, Y.; Ochiai, T.; Fukuzaki, N.; Tajiri, T.; Wakisaka, T. Analysis of flow around robotic fish by three-dimensional fluid-structure interaction simulation and evaluation of propulsive performance. J. Aero Aqua Bio-Mech. 2013, 3, 57-64. [CrossRef]

18. van Rees, W.M.; Gazzola, M.; Koumoutsakos, P. Optimal shapes for anguilliform swimmers at intermediate Reynolds numbers. J. Fluid Mech. 2013, 722, R3. [CrossRef]

19. Van Rees, W.M.; Gazzola, M.; Koumoutsakos, P. Optimal morphokinematics for undulatory swimmers at intermediate Reynolds numbers. J. Fluid Mech. 2015, 775, 178-188.

20. Stefanini, C.; Orofino, S.; Manfredi, L.; Mintchev, S.; Marrazza, S.; Assaf, T.; Capantini, L.; Sinibaldi, E.; Grillner, S.; Wallén, P.; et al. A novel autonomous, bioinspired swimming robot developed by neuroscientists and bioengineers. Bioinspiration Biomimetics 2012, 7, 025001. [CrossRef] 
21. Shriyam, S.; Agrawal, A.; Behera, L.; Saxena, A. Robotic fish design and control based on Biomechanics. IFAC Proc. Vol. 2014, 47, 662-669. [CrossRef]

22. Li, T.; Li, G.; Liang, Y.; Cheng, T.; Dai, J.; Yang, X.; Liu, B.; Zeng, Z.; Huang, Z.; Luo, Y.; et al. Fast-moving soft electronic fish. Sci. Adv. 2017, 3, e1602045. [CrossRef] [PubMed]

23. Scaradozzi, D.; Palmieri, G.; Costa, D.; Pinelli, A. BCF swimming locomotion for autonomous underwater robots: A review and a novel solution to improve control and efficiency. Ocean Eng. 2017, 130, 437-453. [CrossRef]

24. Weihs, D. Hydromechanics of fish schooling. Nature 1973, 241, 290-291. [CrossRef]

25. Novati, G.; Verma, S.; Alexeev, D.; Rossinelli, D.; van Rees, W.M.; Koumoutsakos, P. Synchronisation through learning for two self-propelled swimmers. Bioinspiration Biomimetics 2017, 12, 036001. [CrossRef] [PubMed]

26. Maertens, A.P.; Gao, A.; Triantafyllou, M.S. Optimal undulatory swimming for a single fish-like body and for a pair of interacting swimmers. J. Fluid Mech. 2017, 813, 301-345. [CrossRef]

27. Ashraf, I.; Bradshaw, H.; Ha, T.T.; Halloy, J.; Godoy-Diana, R.; Thiria, B. Simple phalanx pattern leads to energy saving in cohesive fish schooling. Proc. Natl. Acad. Sci. USA 2017, 114, 9599-9604. [CrossRef]

28. Verma, S.; Novati, G.; Koumoutsakos, P. Efficient collective swimming by harnessing vortices through deep reinforcement learning. Proc. Natl. Acad. Sci. USA 2018, 115, 5849-5854. [CrossRef] [PubMed]

29. Dai, L.; He, G.; Zhang, X.; Zhang, X. Stable formations of self-propelled fish-like swimmers induced by hydrodynamic interactions. J. R. Soc. Interface 2018, 15, 20180490. [CrossRef]

30. Park, S.G.; Sung, H.J. Hydrodynamics of flexible fins propelled in tandem, diagonal, triangular and diamond configurations. J. Fluid Mech. 2018, 840, 154-189. [CrossRef]

31. Li, C.; Yang, W.; Xu, X.; Wang, J.; Wang, M.; Xu, L. Numerical investigation of fish exploiting vortices based on the Kármán gaiting model. Ocean Eng. 2017, 140, 7-18. [CrossRef]

32. Jacobsen, N.G.; Fuhrman, D.R.; Fredsøe, J. A wave generation toolbox for the open-source CFD library: OpenFoam R. Int. J. Numer. Methods Fluids 2012, 70, 1073-1088. [CrossRef]

33. Chen, Y.; Xue, M.A. Numerical Simulation of Liquid Sloshing with Different Filling Levels Using OpenFOAM and Experimental Validation. Water 2018, 10, 1752. [CrossRef]

34. Lin, Z.; Yang, W.; Zhou, H.; Xu, X.; Sun, L.; Zhang, Y.; Tang, Y. Communication Optimization for Multiphase Flow Solver in the Library of OpenFOAM. Water 2018, 10, 1461. [CrossRef]

35. Li, C.; Xu, X.; Wang, J.; Xu, L.; Ye, S.; Yang, X. A parallel multiselection greedy method for the radial basis function-based mesh deformation. Int. J. Numer. Methods Eng. 2018, 113, 1561-1588. [CrossRef]

36. Zhao, R.; Li, C.; Guo, X.; Fan, S.; Wang, Y.; Yang, C. A Block Iteration with Parallelization Method for the Greedy Selection in Radial Basis Functions Based Mesh Deformation. Appl. Sci. 2019, 9, 1141. [CrossRef]

37. Kern, S.; Koumoutsakos, P. Simulations of optimized anguilliform swimming. J. Exp. Biol. 2006, 209, 4841-4857. [CrossRef] [PubMed]

38. Gray, J. Studies in Animal Locomotion. J. Exp. Biol. 1933, 15, 506-517.

39. Carling, J.; Williams, T.L.; Bowtell, G. Self-propelled anguilliform swimming: Simultaneous solution of the two-dimensional Navier-Stokes equations and Newton's laws of motion. J. Exp. Biol. 1998, 201, 3143-3166.

40. Donea, J.; Huerta, A.; Ponthot, J.P.; Rodriguez-Ferran, A. Arbitrary Lagrangian-Eulerian Methods. In Encyclopedia of Computational Mechanics; John Wiley and Sons, Ltd.: Hoboken, NJ, USA, 2004; Chapter 14, Volume 1.

41. Hirt, C.; Amsden, A.; Cook, J. An arbitrary Lagrangian-Eulerian computing method for all flow speeds. J. Comput. Phys. 1997, 135, 203-216. [CrossRef]

42. Sahin, M.; Mohseni, K. An arbitrary Lagrangian-Eulerian formulation for the numerical simulation of flow patterns generated by the hydromedusa Aequorea victoria. J. Comput. Phys. 2009, 228, 4588-4605. [CrossRef]

43. Issa, R.I. Solution of the implicitly discretised fluid flow equations by operator-splitting. J. Comput. Phys. 1986, 62, 40-65. [CrossRef]

44. Borazjani, I.; Sotiropoulos, F. Numerical investigation of the hydrodynamics of carangiform swimming in the transitional and inertial flow regimes. J. Exp. Biol. 2008, 211, 1541-1558. [CrossRef] [PubMed]

45. Borazjani, I.; Sotiropoulos, F. Numerical investigation of the hydrodynamics of anguilliform swimming in the transitional and inertial flow regimes. J. Exp. Biol. 2009, 212, 576-592. [CrossRef] 
46. Hieber, S.E.; Koumoutsakos, P. An immersed boundary method for smoothed particle hydrodynamics of self-propelled swimmers. J. Comput. Phys. 2008, 227, 8636-8654. [CrossRef]

47. Ashraf, I.; Godoy-Diana, R.; Halloy, J.; Collignon, B.; Thiria, B. Synchronization and collective swimming patterns in fish (Hemigrammus bleheri). J. R. Soc. Interface 2016, 13, 20160734. [CrossRef]

(C) 2019 by the authors. Licensee MDPI, Basel, Switzerland. This article is an open access article distributed under the terms and conditions of the Creative Commons Attribution (CC BY) license (http:/ / creativecommons.org/licenses/by/4.0/). 